8

\title{
White Dwarf Crystallization
}

\author{
ENRIQUE GARCIA-BERRO
}

Departamento de Física Aplicada. Universidad Politécnica de Cataluña, Jordi Girona Salgado 91, 08094 Barcelona, Spain

\section{MARGARIDA HERNANZ}

Centre d'Estudis Avançats de Blanes, C.S.I.C., Cami de Santa Bárbara, 17900

Blanes, Spain

\begin{abstract}
The inclusion of a detailed treatment of solidification processes in the cooling theory of carbon-oxygen white dwarfs is of crucial importance for the determination of their luminosity function. Carbon-oxygen separation at crystallization yields delays larger than $2 \mathrm{Gyr}$ to cool down to luminosities corresponding to the observed cut-off. This leads to estimates of the age of the galactic disk 1.5 to $2 \mathrm{Gyr}$ older than the ones obtained in previous studies (about $9 \mathrm{Gyr}$ ). Furthermore, the presence of minor chemical species, in particular ${ }^{22} \mathrm{Ne}$, alters significantly the crystallization process, and produces extra delays of 2 to 3 gigayears. However, the detailed computation of the theoretical white dwarf luminosity function, taking into account a reasonable model of galactic chemical evolution, and including the effect of these species, shows that the location of the cut-off, and then the estimated age of the disk, is not modified significantly.

Le traitement détaillé du processus de solidification revêt une importance cruciale dans l'étude du refroidissement des naines blanches carbone-oxygène et la détermination de leur fonction de luminosité. La séparation du carbone et de l'oxygène lors de la cristallisation introduit un retard de plus de $210^{9}$ ans pour atteindre les valeurs de la luminosité correspondant au cut-off observé. Ceci conduit à une estimation de l'âge du disque de 1.5 à $210^{9}$ ans plus vieille que celles obtenues dans les études précédentes. De plus, la présence d'impuretés, en particulier le ${ }^{22} \mathrm{Ne}$, modifie sensiblement le
\end{abstract}


processus de cristallisation et rajoute un délai de 2 à $310^{9}$ ans. Cependant, un calcul détaillé de la fonction de luminosité, prenant en compte un modèle raisonnable d'évolution galactique, et incluant l'effet des impuretés, montre que la position du cut-off, et donc la valeur estimée de l'âge du disque ne sont pas beaucoup modifiées.

\subsection{Introduction}

White dwarfs are remnants of stellar evolution. They are relatively well known objects and their evolutionary time scales are rather large. All these characteristics allow us to consider white dwarfs as fossil stars, so we can use them to do some sort of galactic archaeology. The fundamental tool for that purpose is the white dwarf luminosity function, that is the number of white dwarfs with bolometric magnitude $M_{\text {bol }}$ per cubic parsec per unit bolometric magnitude.

The most reliable observational white dwarf luminosity function is that of Liebert et al (1988). The slope of the increasing part of the white dwarf luminosity function can be roughly explained in terms of a simple model the so-called Mestel law (Mestel, 1952). However, the most relevant feature of the observational white dwarf luminosity function is the pronounced cutoff at $\log \left(L / L_{\odot}\right) \approx-4.5$ (Liebert et al, 1988). There is strong evidence that this effect is not due to the incompleteness of the sample considered or to selection effects (Liebert et al, 1988). More evidence, although indirect, has come recently from the results of Monet et al (1992) who have been able to determine accurate parallaxes and visual magnitudes for a sample of faint red stars. Although this survey is not systematic, it seems quite clear from their results that the cut-off is of intrinsic nature and that there is a significant absence of white dwarfs with luminosities below that of the cut-off. On the other hand, and from a theoretical point of view, it seems clear enough that the drop-off in the white dwarf luminosity function is a consequence of the finite age of the galactic disk, or at least of the solar neighborhood, as pointed out by Winget et al (1987) and Garáa-Berro et al (1988a, 1988b).

However, the computation of theoretical white dwarf luminosity functions requires an accurate white dwarf cooling theory. In fact, the position of the cut-off depends essentially on the cooling time scales of white dwarfs, whereas some aspects of galactic evolution, such as the star formation rate or the initial mass function, affect only the shape of the luminosity function around its maximum (Yuan, 1989, 1992, Iben and Laughlin, 1989) and not the position of the cut-off, unless extremely unrealistic assumptions are 
made. Since the pioneering work of Mestel (1952) the cooling theory of white dwarfs has received continuous interest. $¿$ From the theoretical point of view, once the complicated behaviour of the envelope is properly taken into account (see the review by Mazzitelli, this volume), the most important phenomenon occuring during white dwarf evolution is crystallization. The importance of Coulomb interactions in dense ionic plasmas which form the cores of white dwarfs was first realized by Kirshnitz (1960), Abrikosov (1960) and Salpeter (1961). The relevant parameter is the dimensionless Coulomb coupling constant, defined as:

$$
\Gamma_{e}=\frac{e^{2}}{a_{e} k_{B} T}=2.27510^{5} \frac{\left(\rho Y_{e}\right)^{1 / 3}}{T}
$$

where

$$
a_{e}=\left(\frac{3}{4 \pi n_{e}}\right)^{1 / 3}
$$

is the inter-electronic distance, $n_{e}$ is the free electron density and $Y_{e}$ is the molar fraction of electrons.

When $\Gamma_{e} \ll 1$ Coulomb interactions are negligible, and when $\Gamma_{e} \approx 10$ crystallization sets in. Two essential physical processes are associated with crystallization. The first one is the release of latent heat and the second one is chemical fractionation in the mixture. Both provide extra energy sources and lengthen the cooling time scale of the star. Although the release of the latent heat has been properly included in the calculations of the energetic balance of cooling white dwarfs for long (Van Horn 1968; Lamb and Van Horn 1975; Iben and Tutukov, 1984, Wood, 1992), the effect of chemical fractionation, though energetically dominant, has been usually ignored. This effect depends strongly on the shape of the phase diagram. Mochkovitch (1983) first examined the consequences of an eutectic phase diagram for the carbon-oxygen mixture, as suggested by Stevenson (1980). The effect was shown to be drastic, because of the large release of gravitational energy associated with pure oxygen deposition at the center of the star. The resulting cooling times and luminosity functions (García-Berro et al, 1988a and b) led to age estimates for the galactic disk as large as 15 Gyr. This stimulated more accurate calculations of carbon-oxygen crystallization diagrams (Barrat, Hansen, Mochkovitch (1988); Ichimaru, Iyetomi and Ogata (1988)), which proved that this diagram is not of an eutectic shape. This implies more modest, though still significant, time delays (Isern et al, 1989b, Hernanz et al, 1990). 
Besides carbon and oxygen, minor chemical species are also present in white dwarf interiors, reflecting the initial metallicity of the parent star. The most important being ${ }^{22} \mathrm{Ne}$, a product of the helium burning of the ${ }^{14} \mathrm{~N}$ left by the CNO cycle. Its distribution throughout the star depends on the mass and metallicity of the progenitor of the white dwarf. The importance of ${ }^{22} \mathrm{Ne}$ crystallization on white dwarf cooling was first pointed out by Isern et al (1991), who computed a preliminary phase diagram and estimated time delays as large as $3 \mathrm{Gyr}$. The potential importance of other minor species like iron (Xu and Van Horn, 1992, Chabrier et al, 1993), also stressed the need for further, more accurate calculations of the crystallization diagrams of these species. The most recent study of the properties of arbitrary binary ionic mixtures has been performed by Segretain and Chabrier (1993).

Nevertheless, the computation of realistic theoretical white dwarf luminosity functions also requires the connection of a model of galactic evolution with the cooling sequences. This, in turn, requires a full treatment of the effects of the mass distribution of white dwarfs, the star formation rate and, specially, the evolution of the abundances of minor chemical species. Our aim is to present a complete, accurate treatment of the effect of the different crystallization processes on the white dwarf luminosity function, in order to extract reliable information about the galactic history.

\subsection{Cooling sequences}

Recently Segretain and Chabrier (1993) calculated the phase diagrams of arbitrary binary ionic mixtures, under conditions encountered in dense stellar plasmas, within the framework of modern density-functional theory (DFT) of freezing. These authors characterized the dependence of the shape of the phase diagram on the charge ratio of the mixture $Z_{1} / Z_{2}$. The crystallization diagram was shown to evolve from a spindle form for $0.72<Z_{1} / Z_{2}<1$, into an azeotropic form for $0.58<Z_{1} / Z_{2}<0.72$, and finally into an eutectic form for $Z_{1} / Z_{2}<0.58$. Results of such calculations for $\mathrm{C} / \mathrm{O}, \mathrm{CO} /{ }^{22} \mathrm{Ne}$ and $\mathrm{CO} /{ }^{56} \mathrm{Fe}$ mixtures under conditions of interest are shown on figure 1 . Note that the diagrams are calculated at constant $P_{e}$, i.e constant $\Gamma_{e}$ for a given $T$, and are expressed in dimensionless units for the temperature, so that they apply to any thermodynamic conditions, as long as the pressure of the system is given by the pressure of a homogeneous, degenerate electron background. Given the tremendous complexity of calculating the true phase diagram of a three-component system, the true ternary mixtures have been approximated by effective binary mixtures, where the nitrogen ion mimics an effective, homogeneous $\mathrm{C} / \mathrm{O}$ mixture with an average charge $Z=7$. 


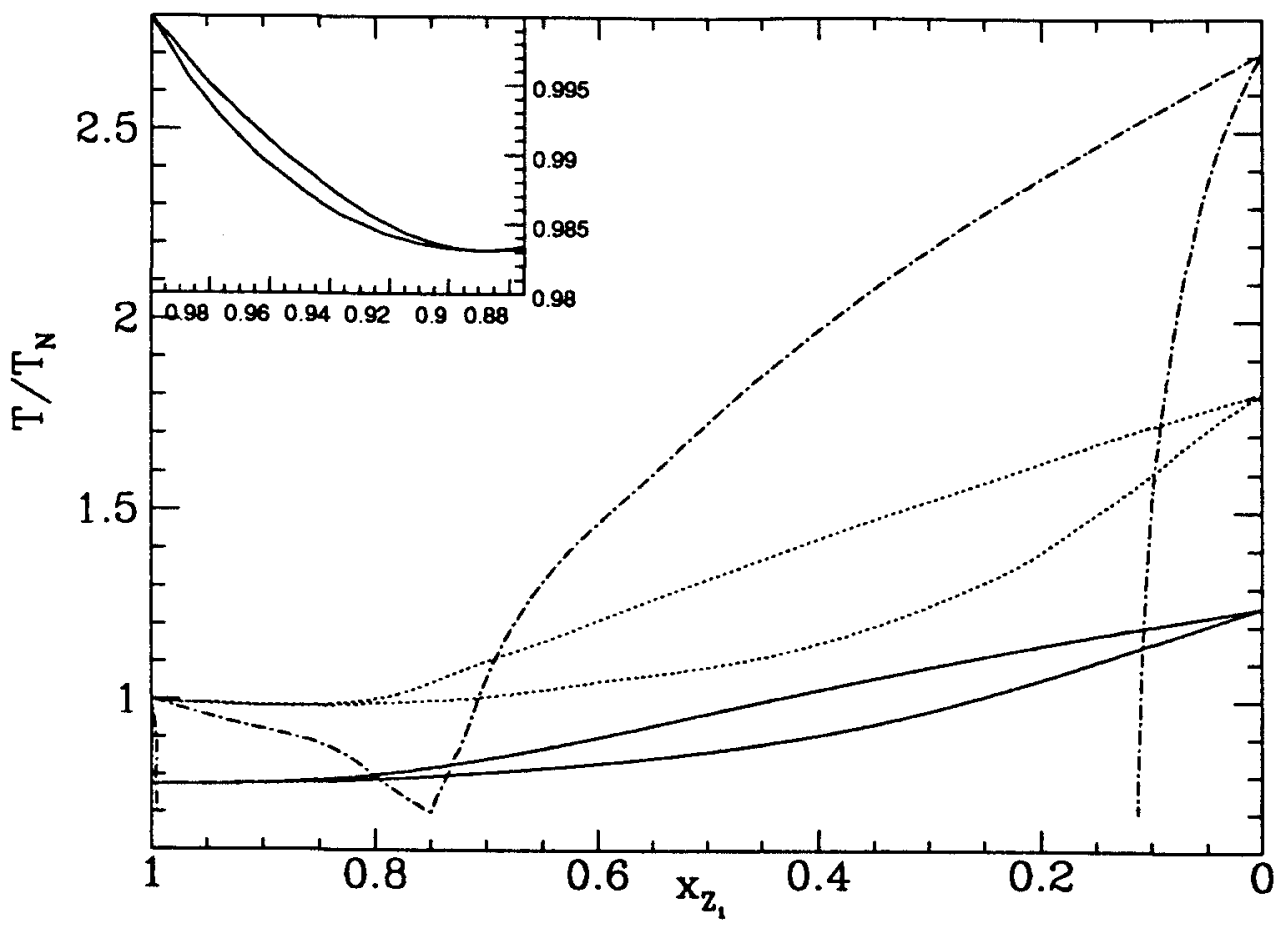

Fig. 8.1 Fluid-solid phase diagrams for a $\mathrm{C} / \mathrm{O}$ mixture (full line), a $\mathrm{N} /{ }^{22} \mathrm{Ne}$ mixture (dotted line) and a $N /{ }^{56} \mathrm{Fe}$ mixture (dotted-dashed line). Temperature is given in units of pure $\mathrm{N}(Z=7)$ crystallization temperature. $Z_{1}$ denotes the smallest charge in each mixture. The insert shows a blow up of the azeotropic phase diagram, left to the azeotropic concentration.

In the case of an azeotropic phase diagram, crystallization proceeds within a particular scheme, as described by Isern et al (1991). The density discontinuity at crystallization is given by (Mochkovitch 1983):

$$
\frac{\Delta \rho}{\rho}=\frac{\rho_{S}-\rho_{L}}{\rho}=-\frac{\Delta P_{\text {nuc }}}{\gamma P_{e}}-\frac{\Delta Y_{e}}{Y_{e}}
$$

where $P_{\text {nuc }}$ denotes the ionic pressure and $\gamma$ is the adiabatic index. In the case of a $\mathrm{N} /{ }^{22} \mathrm{Ne}$ mixture, the abundance of ${ }^{22} \mathrm{Ne}$ is smaller than the azeotropic concentration, so that $\Delta Y_{e} / Y_{e}$ is larger than $\Delta P_{n u c} / \gamma P_{e}$, and then $\Delta \rho<0$. The solid, less dense than the surrounding liquid, will rise towards the surface, leaving behind a denser, ${ }^{22} \mathrm{Ne}$-enriched liquid. As the solid floats upwards, it melts again, so that the overlying liquid develops a composition gradient. This process continues until the azeotropic concentration is reached, where a solid with this density freezes. At this stage, all ${ }^{22} \mathrm{Ne}$ has been collected into the core. The same process is also valid for ${ }^{56} \mathrm{Fe}$. 


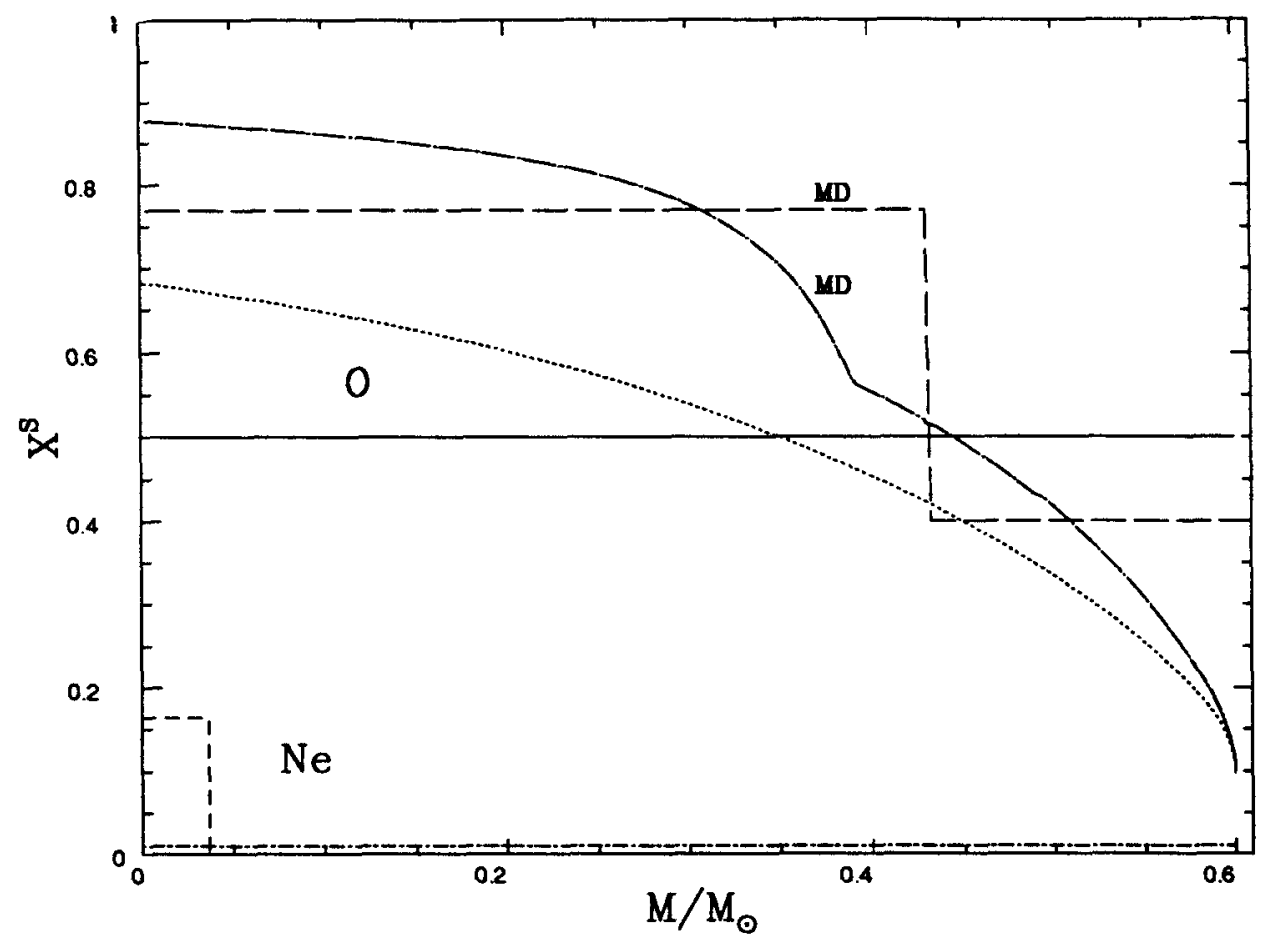

Fig. 8.2 Density profiles for $\mathrm{O}$ (top) and ${ }^{22} \mathrm{Ne}$ (bottom) obtained either from the true phase diagrams (dotted line and dashed line) or from the assumption of no chemical fractionation at crystallization (full line and dot-dashed line). The curves labeled MD show the oxygen density profile with (dot-dashed line) and without (dashed line) chemical fractionation at crystallization when the initial $\mathrm{C} / \mathrm{O}$ distribution is given by the stratified profiles of Mazitelli and D'Antona (1986).

This process will no longer occur above a maximum oxygen concentration in the solid, for which eqn. (2) yields $\Delta \rho>0$, no matter the shape of the phase diagram. For a neon and an oxygen mass concentration in the liquid $X_{{ }_{22 \mathrm{Ne}}}=1 \%$ and $\left[X_{O}\right]_{L}=50 \%$, the adopted phase diagram leads to an upper limit for the oxygen mass concentration in the solid $\left(\left[X_{O}\right]_{s}\right)_{\max }=$ $62 \%$. As shown on figure $1,{ }^{22} \mathrm{Ne}$ will crystallize first, in an effective $\mathrm{CO}$ mixture. All neon will deposit at the center of the star at the azeotropic concentration $x_{22} \mathrm{Ne} \approx 11 \%$, following the afore-described scenario. Figure 2 shows the composition profiles for $\mathrm{O}$ and ${ }^{22} \mathrm{Ne}$ obtained with the diagrams shown on figure 1 .

We have included the previous results in the calculation of the cooling time of white dwarfs. In order to avoid the complicated behavior of hot white dwarfs we will study the evolution of white dwarfs starting from luminosities of order $\sim 10^{-1} L_{\odot}$, corresponding to core temperatures of the order of $\sim 6 \times 10^{7} \mathrm{~K}$. Under these conditions neutrino losses can be 
neglected. Thermonuclear reactions in the outer layers are also unimportant (Iben and Tutukov, 1984). Also, central densities of most single white dwarfs are not high enough to allow pycnonuclear reactions to occur, so we can neglect this source of energy as well. Hence, the star luminosity arises only from the thermal and gravitational energy release:

$$
L(T)=\frac{d B}{d t}
$$

where $B=U+\Omega$ is the so-called binding energy, being $U$ and $\Omega$ the thermal and gravitational energy content of the white dwarf which are given, respectively, by the expressions

$$
\Omega=-\int_{0}^{M_{W D}} G \frac{m}{r} d m \text { and } U=\int_{0}^{M_{W D}} u d m
$$

The crystallization of each layer, and the related latent heat and gravitational energy release due to chemical differentiation, are taken into account automatically as the temperature of the star decreases along evolution.

On the other hand, a relation between the luminosity of the star and the temperature of its isothermal core is necessary to avoid the difficulties inherent to the isolating, semidegenerate envelope of the white dwarf. Several of such relations have been obtained for a C-rich envelope (Lamb and Van Horn, 1975), a He-rich envelope (Wood and Winget, 1989) and a H-rich envelope (D'Antona and Mazzitelli, 1989). We have chosen the following functional dependence

$$
\frac{L(T)}{L_{\odot}}=\frac{M_{W D}}{M_{\odot}} F(T)
$$

which takes into account the leading term of the mass dependence of the luminosity and it is enough for our purposes. In the case of a He envelope, the results of Wood and Winget (1989) for a $0.6 \mathrm{M}_{\odot}$ white dwarf (their CO60400 sequence) can be fitted by:

$$
\begin{aligned}
\log F(T)= & 1.98695\left(\log T_{6}\right)^{5}-8.15521\left(\log T_{6}\right)^{4}+ \\
& 11.863\left(\log T_{6}\right)^{3}-6.88515\left(\log T_{6}\right)^{2}+ \\
& 3.16893\left(\log T_{6}\right)-4.56649
\end{aligned}
$$


The time required to cool down to a temperature $T$, corresponding to a luminosity $L(T)$, is then given by:

$$
t_{\text {cool }}(L)=\int_{T_{0}}^{T} \frac{d B}{L}
$$

and the characteristic cooling time $\tau_{\text {cool }}=d t_{\text {cool }} / d M_{\text {bol }}$ reads

$$
\tau_{c o o l}=-0.4 \frac{d B}{d T} \frac{d T}{d L}
$$

from this expression it is quite clear that the core feeds the cooling through the release of binding energy whereas the envelope controls it according to the dependence of the luminosity on the temperature of the isothermal core.

In order to characterize the effect on white dwarf cooling of the different crystallization processes, we compare the different calculations with a reference cooling time, obtained when a $\mathrm{C} / \mathrm{O}$ white dwarf in which there are not trace elements present is forced to crystallize with no chemical fractionation. The different calculations include: case (i) a pure $\mathrm{C} / \mathrm{O}$ white dwarf with fractionation, and case (ii) a CO/ ${ }^{22} \mathrm{Ne}$ white dwarf with $X_{22 \mathrm{Ne}}=1.0 \%$.

We consider first a $0.6 M_{\odot}$ white dwarf with a uniform equimassive $\left(X_{\mathrm{C}}=\right.$ $\left.X_{\mathrm{O}}=0.50\right) \mathrm{C} / \mathrm{O}$ distribution in the fluid. The importance of an initial composition gradient in the fluid phase will be considered later. The different results are displayed in figures 3 and 4 . The sudden crystallization of ${ }^{22} \mathrm{Ne}$ at the azeotropic concentration is reflected by the abrupt release of gravitational energy at the crystallization temperature (luminosity). This energy source will sustain the star at the same luminosity for a considerable amount of time. Crystallization of $\mathrm{C} / \mathrm{O}$ occurs through a more continuous process.

Figure 3 shows the binding energy obtained when the gravitational energy released respectively by $\mathrm{C} / \mathrm{O}$ crystallization, and $\mathrm{CO} /{ }^{22} \mathrm{Ne}$ crystallization, is taken into account. The amounts of gravitational energy released between $t_{c}$ and $t_{-4.5}$, where $t_{c}$ is the time where crystallization occurs, and $t_{-4.5}$ is the time where the star reaches the cut-off luminosity $L=10^{-4.5} L_{\odot}$, are respectively $\Delta B_{\mathrm{C} / \mathrm{O}}=2.3510^{46} \mathrm{erg}$, and $\Delta B_{\mathrm{N} /{ }^{22} \mathrm{Ne}}=7.1310^{46} \mathrm{erg}$. Onset of crystallization at the center of the star is found to occur respectively at $T_{\mathrm{C} / \mathrm{O}}=3.5910^{6} \mathrm{~K}$, and $T_{\mathrm{N} /{ }^{22} \mathrm{Ne}}=3.8410^{6} \mathrm{~K}$. Thus the energy release due to the differentiation of the minor element ${ }^{22} \mathrm{Ne}$ is of the same order as the one due to the crystallization of the major constituents, $\mathrm{C}$ and $\mathrm{O}$.

The effect on the cooling time of a $0.6 M_{\odot}$ white dwarf is shown in figure 4. The crystallization of $1 \%$ of neon occurs at a higher luminosity, and produces a larger time delay than the crystallization of $\mathrm{C} / \mathrm{O}$. Time delays 


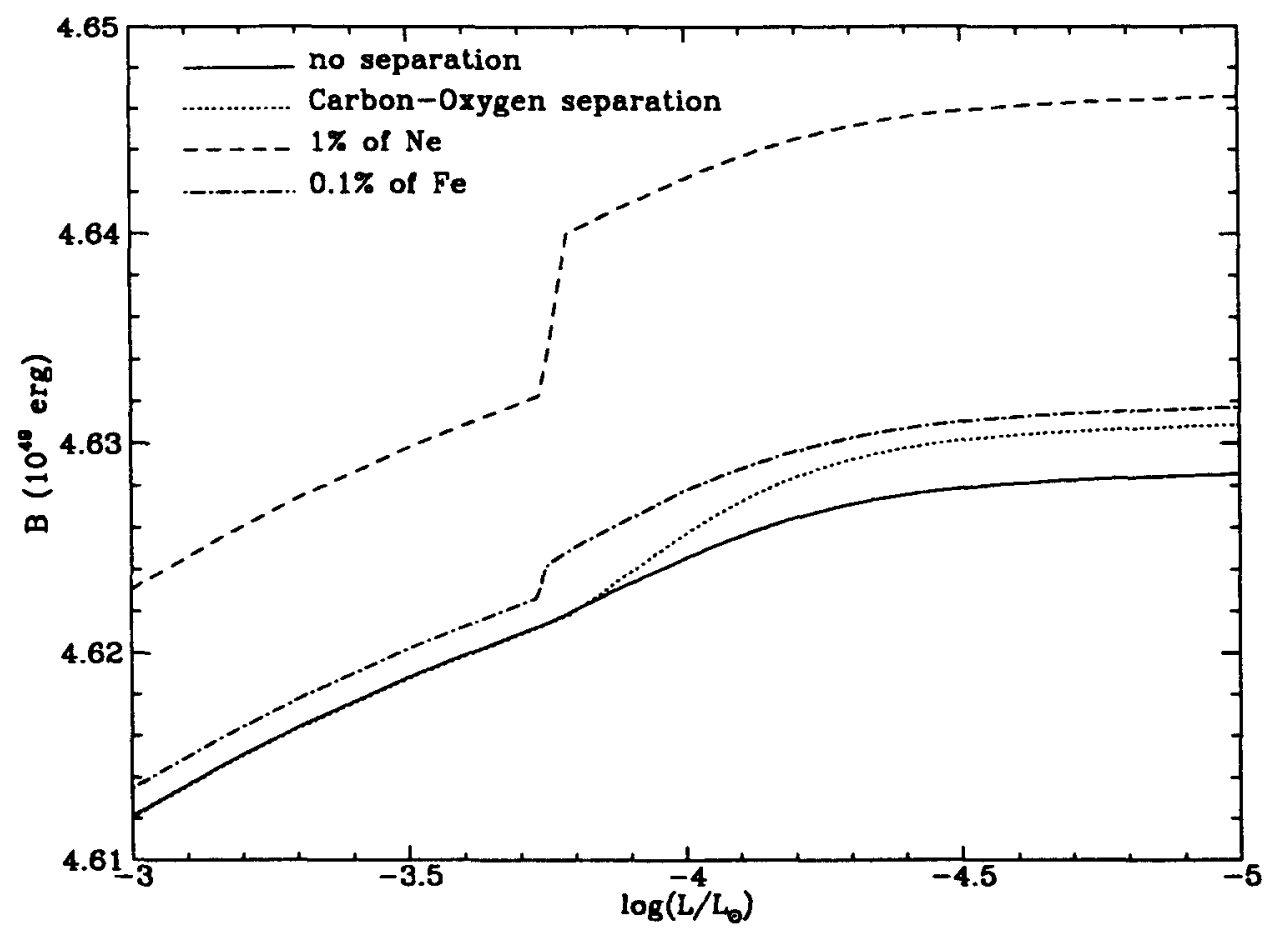

Fig. 8.3 Binding energy of a $\mathrm{C} / \mathrm{O}$ star (dotted line), a $\mathrm{CO} /{ }^{22} \mathrm{Ne}$ star (dashed line) and a $\mathrm{CO} /{ }^{56} \mathrm{Fe}$ star (dotted-dashed line). Energy released by crystallization is shown by the kinks. The full line corresponds to the case with no fractionation at crystallization.

produced by crystallization of $\mathrm{C} / \mathrm{O}$ and ${ }^{22} \mathrm{Ne}$ to reach $L=10^{-4.5} L_{\odot}$ are respectively $2.2 \mathrm{Gyr}$, and $3.4 \mathrm{Gyr}$. For $\mathrm{C} / \mathrm{O}$, our result is about a factor 2 larger than previous estimates (Barrat et al 1988; Ichimaru et al 1988), and stems from the larger density discontinuity at crystallization obtained with our phase diagram. Enrichment of the core by trace elements is sufficient to extend the cooling time by an amount comparable to that produced by $\mathrm{C} / \mathrm{O}$ chemical fractionation, about $20 \%$ of the time obtained when ignoring chemical separation at crystallization (Wood, 1992). We note from figure 4 that the time delay produced by the crystallization of ${ }^{22} \mathrm{Ne}$ and the remaining $\mathrm{C} / 0$ mixture (dotted-dashed line) gives $5.35 \mathrm{Gyr}$ at $L=10^{-4.5} L_{\odot}$, almost exactly the sum of the time delays obtained when treating separately ${ }^{22} \mathrm{Ne}$ crystallization (short-dashed line) and crystallization of the pure C/O mixture with no trace elements (dotted line), which gives $2.2+3.4=5.6 \mathrm{Gyr}$. This result has been confirmed for different concentrations of ${ }^{22} \mathrm{Ne}$.

In figure 5, we show the characteristic cooling times for our reference model as a function of luminosity for white dwarfs within a mass range 0.5 $\mathrm{M}_{\odot}$ to $1.2 \mathrm{M}_{\odot}$. High mass white dwarfs crystallize at higher luminosities, thus having smaller time delays, and then reach the Debye cooling regime 


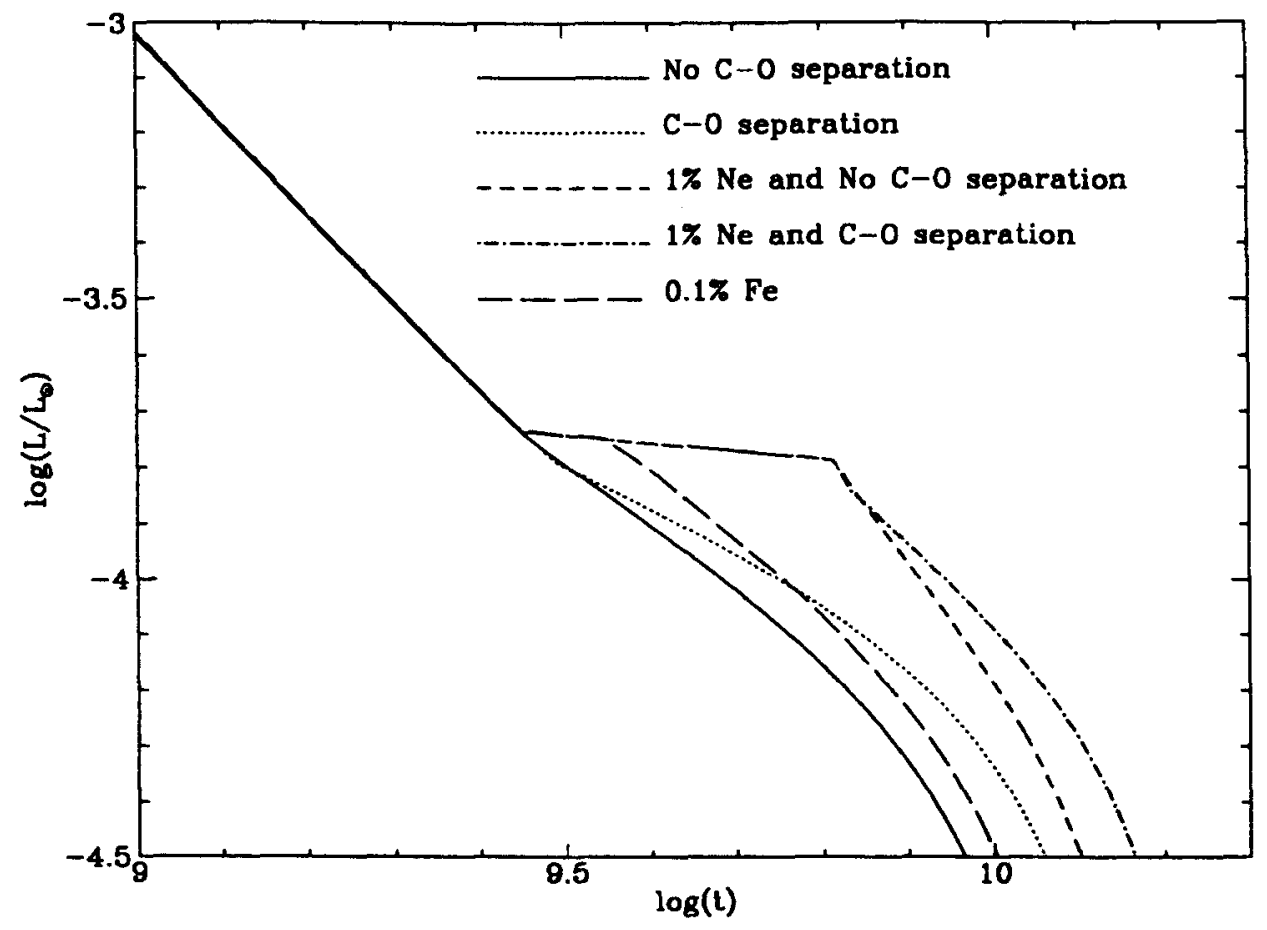

Fig. 8.4 Cooling time of a $\mathrm{C} / \mathrm{O}$ star (dotted line), a $\mathrm{CO} /{ }^{22} \mathrm{Ne}$ star with $X_{{ }^{22} \mathrm{Ne}}=0.01$ (short-dashed line), and a CO/ $/{ }^{22} \mathrm{Ne}$ star where the remaining $\mathrm{C} / \mathrm{O}$ mixture crystallizes after ${ }^{22} \mathrm{Ne}$ has crystallized (dot-dashed line). The full line corresponds to the reference model, when the $\mathrm{C} / \mathrm{O}$ star is forced to crystallize with no chemical separation.

before, thus cooling down faster. The characteristic cooling times for case (i) are shown in figure 6 . The large bumps reflect the important release of gravitational energy at the crystallization luminosity.

In figure 7, we compare the characteristic cooling times of a $0.6 \mathrm{M}_{\odot}$ white dwarf with ${ }^{22} \mathrm{Ne}$ abundances by mass $X_{22 \mathrm{Ne}}=0.01$ and 0.03 , case (ii), with the ones obtained for a white dwarf without neon, i.e. $X_{22} \mathrm{Ne}=0$. The peaks in the characteristic cooling times reflect the sudden release of gravitational energy associated with neon crystallization, which occurs over a very narrow temperature (luminosity) range. This is due to the fact that the azeotropic point of the phase diagram is only slightly depressed (see Segretain et al, 1993). The different positions of the peaks correspond to the different temperatures (luminosities) at which crystallization starts, according to the phase diagram.

We now consider an initial composition gradient in the $\mathrm{C} / \mathrm{O}$ distribution inside the star, resulting from previous evolutionary stages. We have performed the same calculations with the $\mathrm{C} / \mathrm{O}$ distribution obtained by Mazzitelli and D'Antona (1986). The reference model is the same as men- 


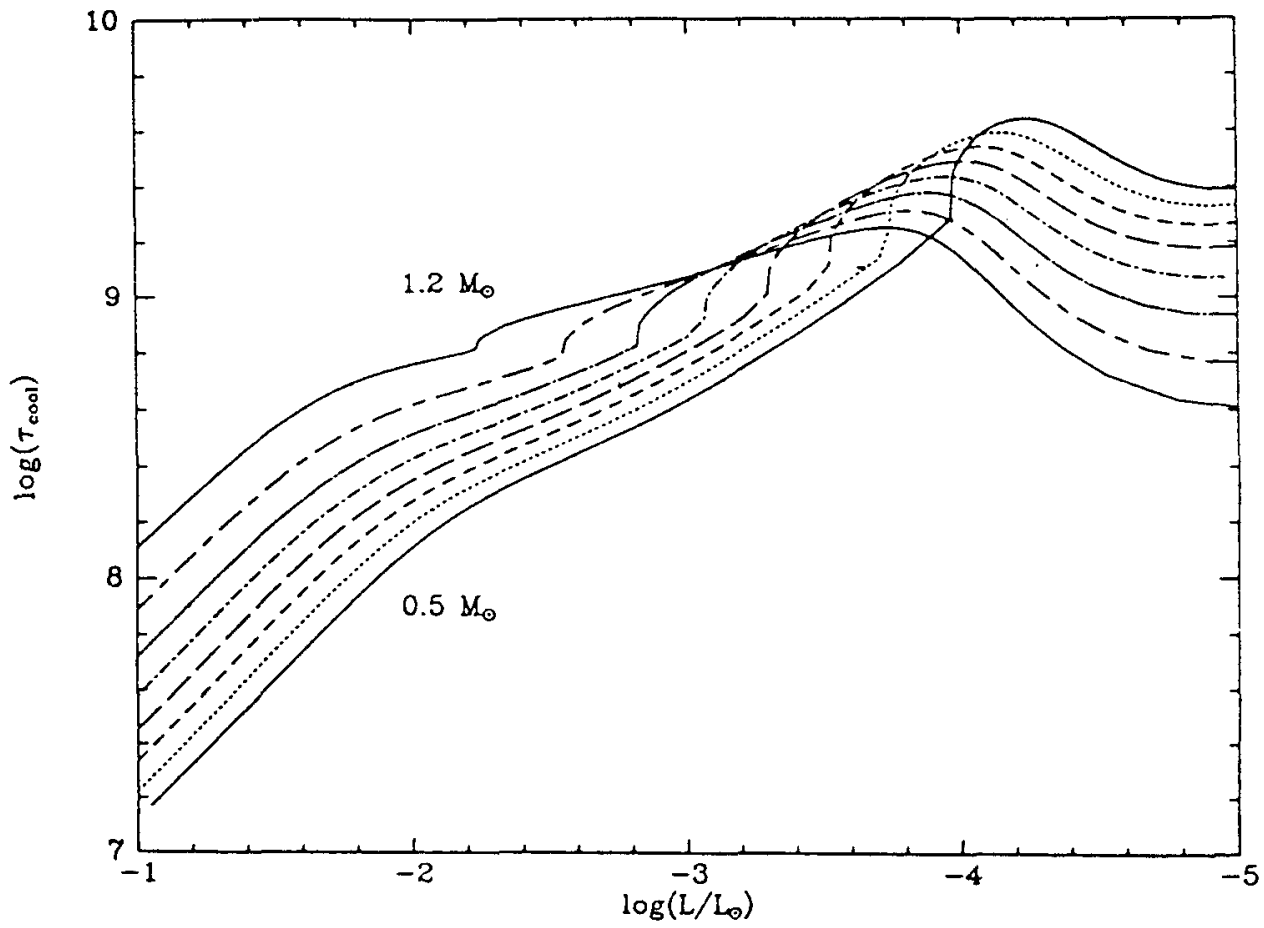

Fig. 8.5 Characteristic cooling times vs luminosity, for $\mathrm{C} / \mathrm{O}$ white dwarfs with masses ranging from 0.5 to $1.2 \mathrm{M}_{\odot}$, computed with the assumption of no fractionation at crystallization.

tioned above, i.e. does not include stratification and chemical separation. The three other models include chemical separation and no stratification, case (ii) already discussed, (iii) stratification and no chemical separation, and (iv) stratification and chemical separation. The results are shown on figure 8. Stratification leads to an oxygen-enriched core, and then to a smaller gravitational energy release at crystallization, and a smaller time delay. Moreover, the latent heat is released at higher temperature (see the $\mathrm{C} / \mathrm{O}$ phase diagram), i.e. at larger luminosity. Note that stratification takes place for $M<1.0 M_{\odot}$ only.

The characteristic cooling times are similar to the ones displayed on figure 5 , except that, because of the higher oxygen abundance at the center of the star, crystallization begins at a higher temperature, and then at a greater luminosity, for masses smaller than $1.0 \mathrm{M}_{\odot}$. These cooling sequences agree with the results of Wood (1992), except that this author does not consider the dependence of the initial $\mathrm{C} / \mathrm{O}$ profile on the mass of the white dwarf. 


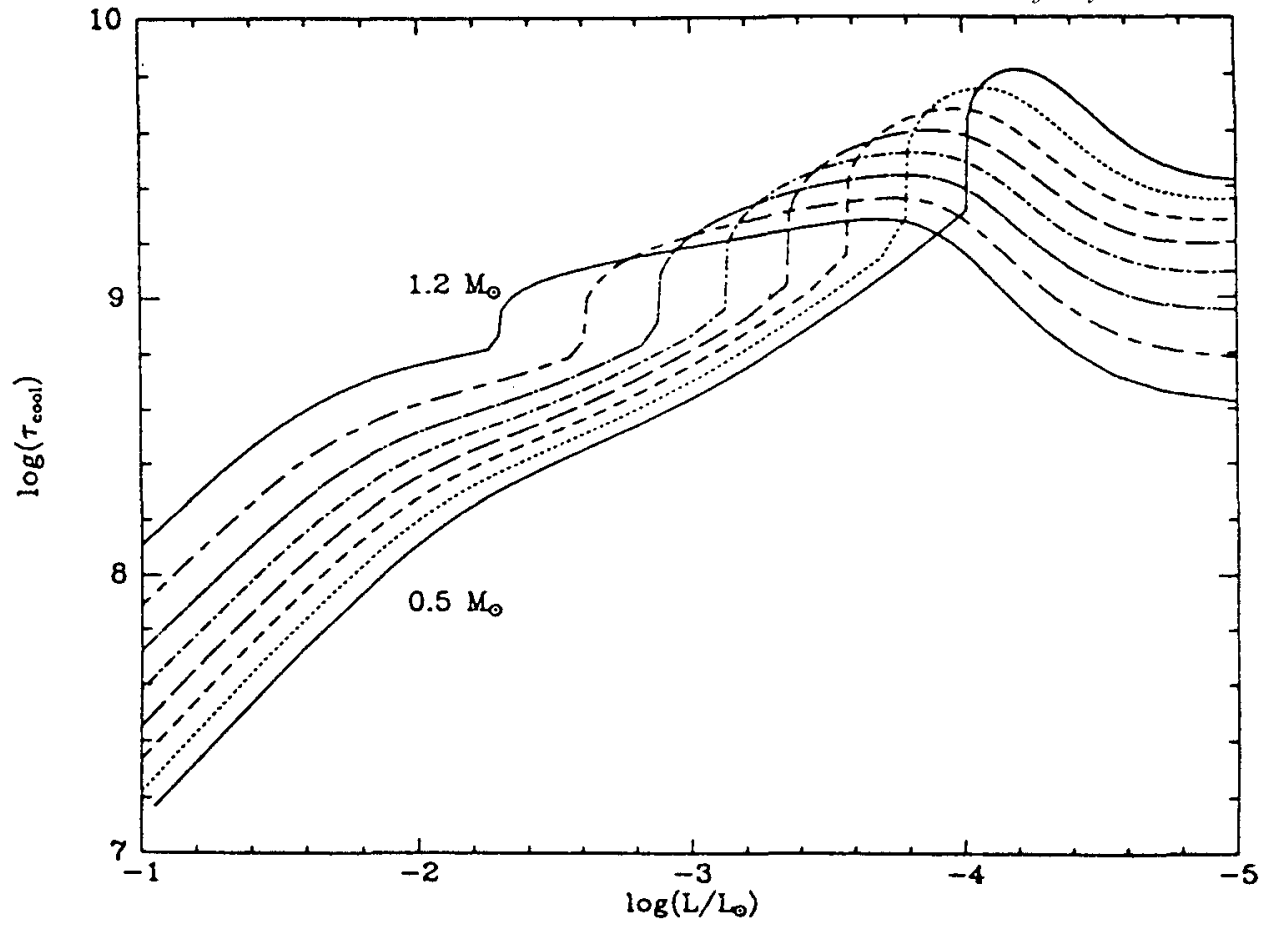

Fig. 8.6 Same as figure 5, but taking into account the effect of carbonoxygen fractionation (case ii), which yields a larger bump at the crystallization luminosity.

\subsection{The luminosity function}

The luminosity function including the effect of minor chemical species has been computed from a generalization of the method developed by Noh and Scalo (1990). The distribution function $f(l, m, Z)$ gives the number of white dwarfs per unit volume in a generalized phase space where the coordinates are the mass, the metallicity and $l=-\log \left(L / L_{\odot}\right)$, being $L$ the luminosity of the white dwarf. This distribution reads:

$$
\begin{aligned}
f(l, m, Z)= & \frac{1}{i(l, m, Z)} \int_{-\infty}^{+\infty} \delta\left(l-l_{0}\right) B_{w d}(t, m, Z) \dot{l}(t) d t \\
& =\frac{1}{i(l, m, Z)} B_{w d}\left(t_{0}, m, Z\right)
\end{aligned}
$$

where $l$ is proportional to the bolometric magnitude, $\delta$ is the delta function, $l_{0}=-\log \left(L_{0} / L_{\odot}\right)$, being $L_{0}$ the white dwarf luminosity at birth (assuming that all white dwarfs were born with the same luminosity), $1 / i(l, m, Z)$ is proportional to the characteristic cooling time. $B_{w d}\left(t_{0}, m, Z\right)$ is the socalled source function for white dwarfs at time $t_{0}$, defined as the total num- 


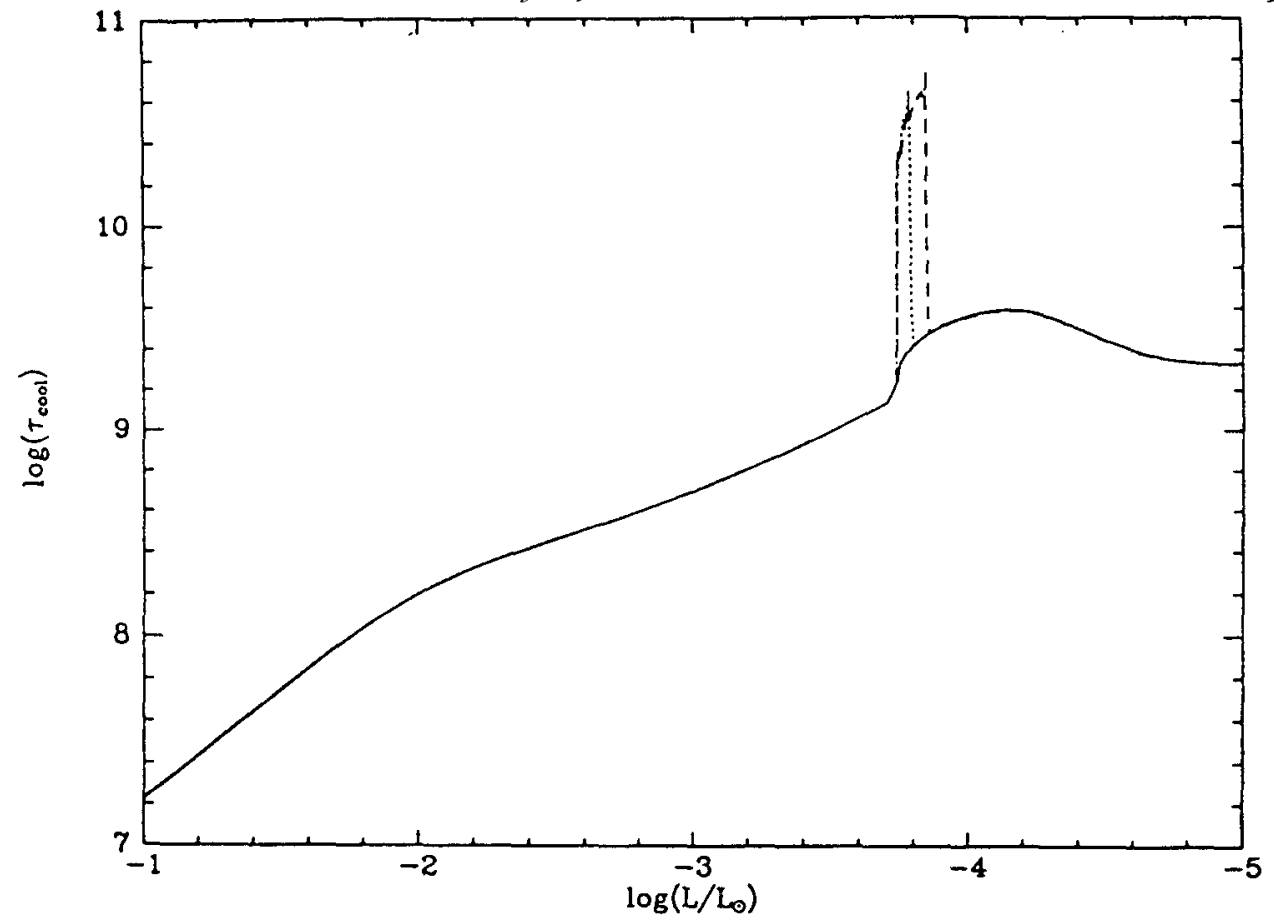

Fig. 8.7 Characteristic cooling times vs luminosity, for a $0.6 \mathrm{M}_{\odot} \mathrm{C} / \mathrm{O}$ white dwarf with $X^{22} \mathrm{Ne}=0 \%$ (solid line), $1 \%$ and $3 \%$ respectively.

ber of white dwarfs with mass $m$ and metallicity $Z$ born at time $t_{0}$, per mass, metallicity and time units. Thus

$$
B_{w d}\left(t_{0}, m, Z\right)=\frac{d^{3} N}{d M d Z d t}=\phi(M) \psi\left(t_{b}\right) \delta\left(Z-Z\left(t_{b}\right)\right)
$$

Here $M$ is the mass of the progenitor of a white dwarf of mass $m, \phi(M)$ the initial mass function, generally assumed to be constant in time, $\psi(t)$ the star formation rate, expressed as mass of stars formed per unit volume $\left(\mathrm{pc}^{3}\right), t_{0}$ is the birth time of the white dwarf (with a luminosity $L_{0}$ which we will consider to be large compared with the actual luminosity, $L_{0} \gg L$ ), and $t_{b}$ is the birth time of the progenitor star, obtained from:

$$
t_{b}+t_{m s}(M)+t_{\text {cool }}\left(L, M, Z\left(t_{b}\right)\right)=t_{d i s k}
$$

where $t_{d i s k}$ is the age of the disk and $t_{m s}$ is the time spent on the main sequence. Note that by adopting this expression for the source function we are implicitly assuming that the metallicity of white dwarfs is that of the interstellar medium when their progenitors were born.

The integration of (7) gives the white dwarf luminosity function $n(L)$, i.e. 


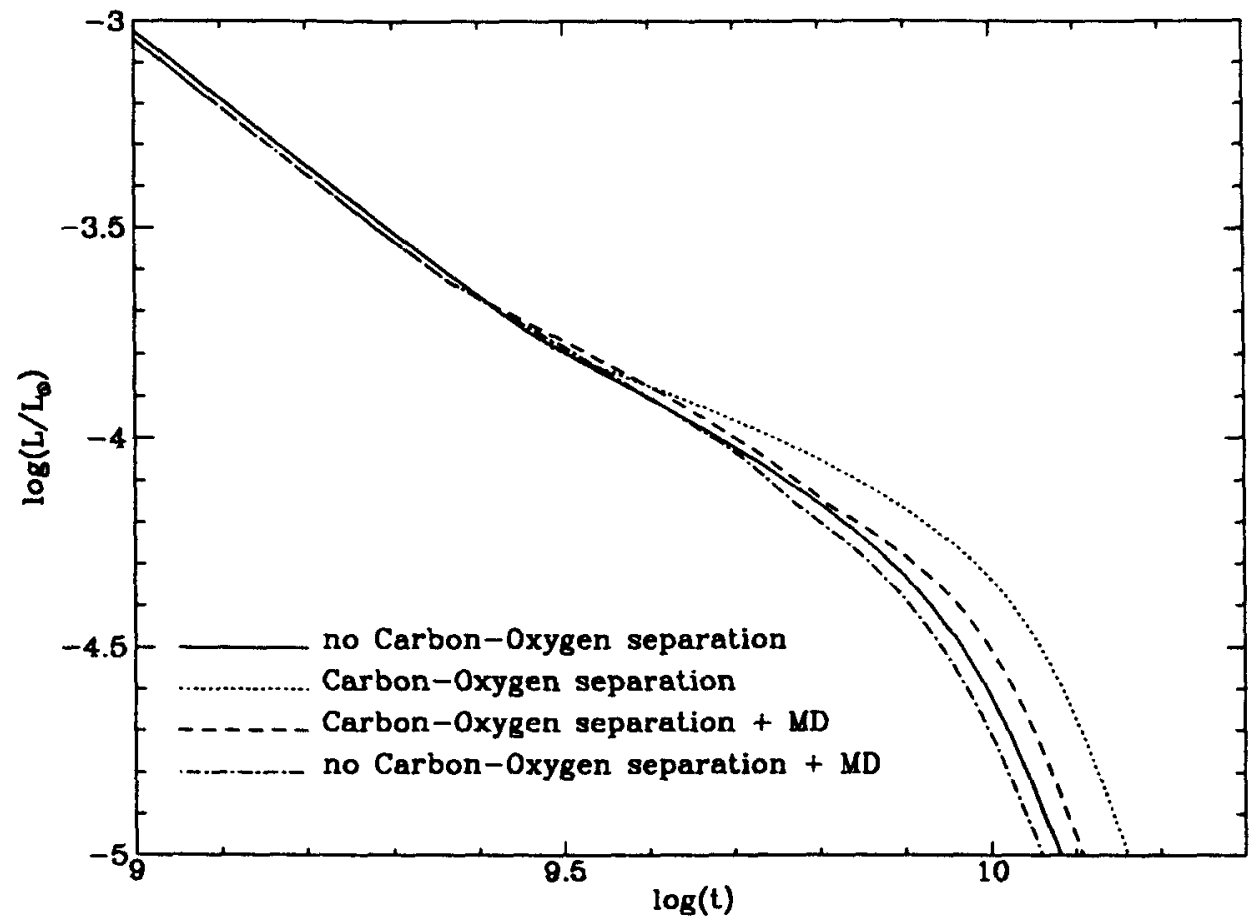

Fig. 8.8 Effect of initial stratification on the cooling time of a C/O $0.6 M_{\odot}$ white dwarf. Full line: no separation, no stratification; dotted line: separation, no stratification; dotted-dashed line: no separation, stratification; dashed line: separation, stratification. MD stands for Mazzitelli and D'Antona (1986).

the number of white dwarfs per unit of bolometric magnitude, per cubic parsec, with luminosity $L$ :

$$
\begin{gathered}
n(L)=\int_{M_{\text {ins }}}^{M_{\text {oup }}} \tau_{\text {cool }}\left(L, M, Z\left(t_{b}\right)\right) \psi\left(t_{\text {disk }}-t_{\text {cool }}\left(L, M, Z\left(t_{b}\right)\right)-\right. \\
\left.t_{m s}(M)\right) \phi(M) d M
\end{gathered}
$$

where $M_{\text {sup }}$ and $M_{\text {in } f}$ denote respectively the maximum and the minimum mass of the white dwarf progenitors which contribute at luminosity $L . M_{\text {inf }}$ is obtained by setting $t_{b}=0$ in expression (8):

$$
t_{m s}\left(M_{\text {inf }}\right)+t_{\text {cool }}\left(L, M_{\text {inf }}, Z_{0}\right)=t_{\text {disk }}
$$

where $Z_{0}$ is the initial $(t=0)$ metallicity of the interstellar medium, usually taken as 0 . If we focus on pure $C / O$ white dwarfs, then eqn. (9) reads 


$$
\begin{gathered}
n(L)=\int_{M_{\text {inf }}(L)}^{M_{\text {sup }}} \tau_{\text {cool }}(L, M) \psi\left(t_{\text {disk }}-t_{\text {cool }}(L, M)-t_{m s}(M)\right) \\
\phi(M) d M
\end{gathered}
$$

where $M_{\text {inf }}$ satisfies now: $t_{m s}\left(M_{\text {inf }}\right)+t_{\text {cool }}\left(L, M_{\text {inf }}\right)=t_{\text {disk }}$.

Conversely, if we focus on the influence of minor chemical species on the white dwarf luminosity function, the cooling rates depend on the mass of the white dwarf, its luminosity and its metal content. For a sake of simplicity, the dependence of cooling times and characteristic cooling times on the mass of the white dwarf will be ignored and calculations will be performed for a typical $M_{*}=0.6 \mathrm{M}_{\odot}$ white dwarf only. This is justified by the weak mass-dependence of $t_{c o o l}$ and $\tau_{\text {cool }}$ when compared with their strong metallicity-dependence. In this case, the luminosity function reads:

$$
\begin{gathered}
n(L)=\int_{M_{\text {inf }}(L)}^{M_{\text {sup }}} \tau_{\text {cool }}\left(L, M_{*}, Z\right) \psi\left(t_{\text {disk }}-t_{\text {cool }}\left(L, M_{*}, Z\right)-\right. \\
\left.t_{m s}(M)\right) \phi(M) d M
\end{gathered}
$$

where $M_{\text {inf }}$ is now given by: $t_{\text {disk }}=t_{\text {cool }}\left(L, M_{*}, Z_{0}\right)+t_{m s}\left(M_{\text {inf }}\right)$. A close look at equation (12) reveals that, in fact, the effect of different progenitor masses is taken into account correctly.

As can be seen from the previous expressions, the computation of the white dwarf luminosity function requires not only accurate cooling sequences, but also a model of galactic evolution. A good standard model for our purposes is that of Clayton (1984), which takes into account the infall of matter over the galaxy for a certain amount of time. The reason of this choice is basically its simplicity. Of course, there are more elaborate models of galactic evolution (see for instance Abia et al, 1991, and Bravo et al, 1993) but for our purposes - that is, showing the importance of a proper treatment of crystallization - this SFR is enough. Concerning the initial mass function, IMF, the one proposed by Salpeter (1961) has been adopted.

We have computed luminosity functions for the cooling sequences described in the previous section. Figures 9 and 10 show the luminosity function (11) for our reference model and case (iii), i.e. no separation at crystallization without and with initial stratification respectively, computed for two different ages of the galactic disk in each case, namely 8.8 and $10.5 \mathrm{Gyr}$ and 8.5 and $10.3 \mathrm{Gyr}$, respectively. These values best reproduce the position of the observed cut-off, obtained with two different approximations for 


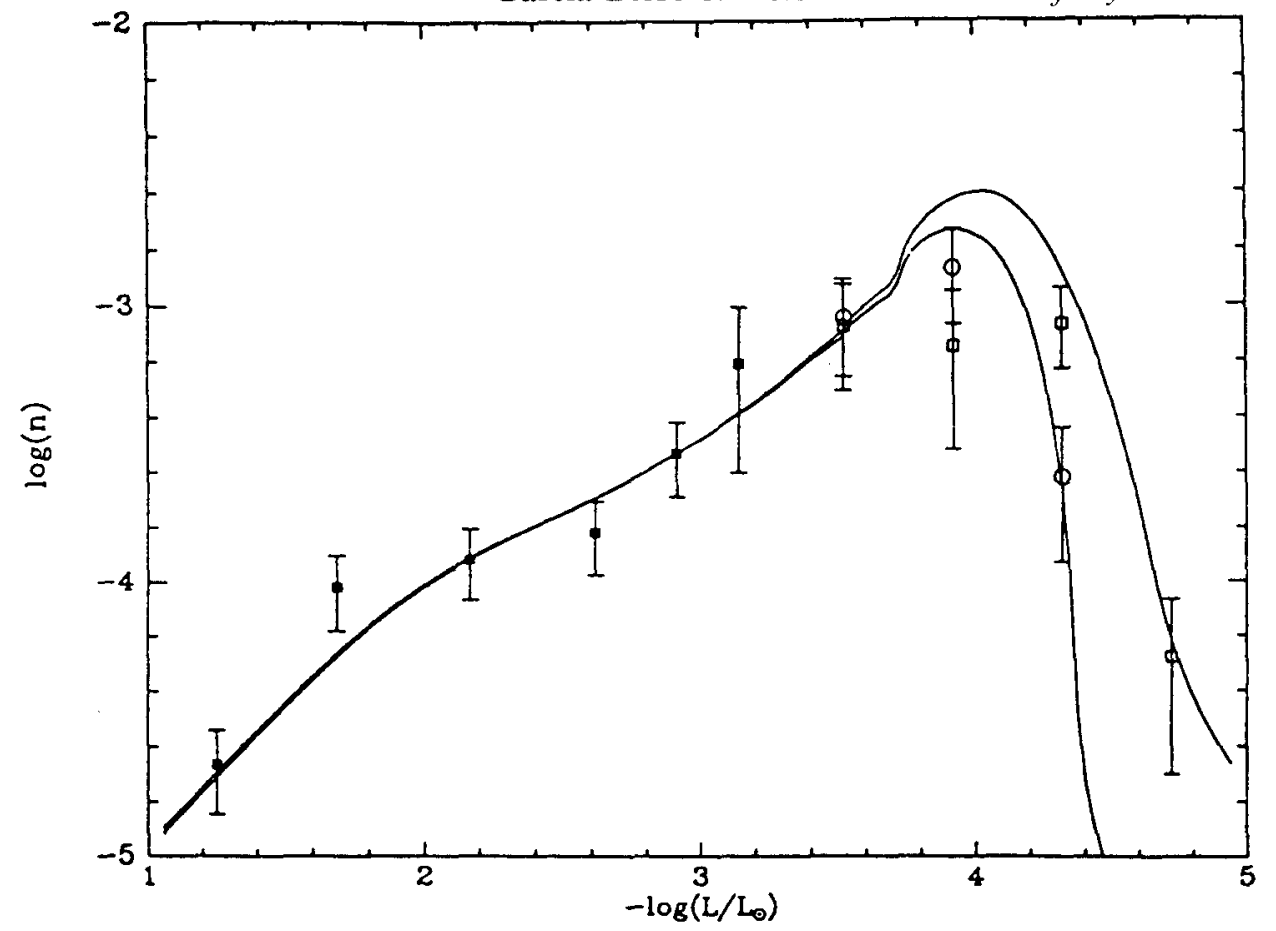

Fig. 8.9 Theoretical luminosity functions for $\mathrm{C} / \mathrm{O}$ white dwarfs with no fractionation (reference model), for ages of the disk $t_{\text {disk }}=8.8$ (left line) and 10.5 Gyr (right line). Solid squares represent the bright end of the luminosity function taken from Fleming, Liebert and Green (1986). For low luminosity objects, two approximations to the bolometric correction are taken, following Liebert, Dahn and Monet (1988): either model BC for cool DAs and blackbody BC for cool non-DAs (open circles), or zero BC for all cool white dwarfs (open squares).

the bolometric correction of the coolest white dwarfs (the bright portion of the observational luminosity function has been taken from Fleming, Liebert and Green, 1986). For low luminosity objects, two approximations to the bolometric correction are considered, following Liebert, Dahn and Monet (1988): either model bolometric corrections for cool DAs and blackbody bolometric corrections for cool non-DAs (open circles), or zero bolometric corrections for all cool white dwarfs (open squares). This dubiousness in the faint end of the observed luminosity function leads to an uncertainty of about $2 \mathrm{Gyr}$ in the age of the disk, as can be seen from the figures. It is important to stress that these two possible observational luminosity functions, are not equivalent to Wood's error boxes approach: curves inside the error boxes do not necessarily cross the error bars, even though the resulting uncertainty in the age of the disk is similar. The small difference between our reference model and case (iii) stems from the effect of initial stratification in 


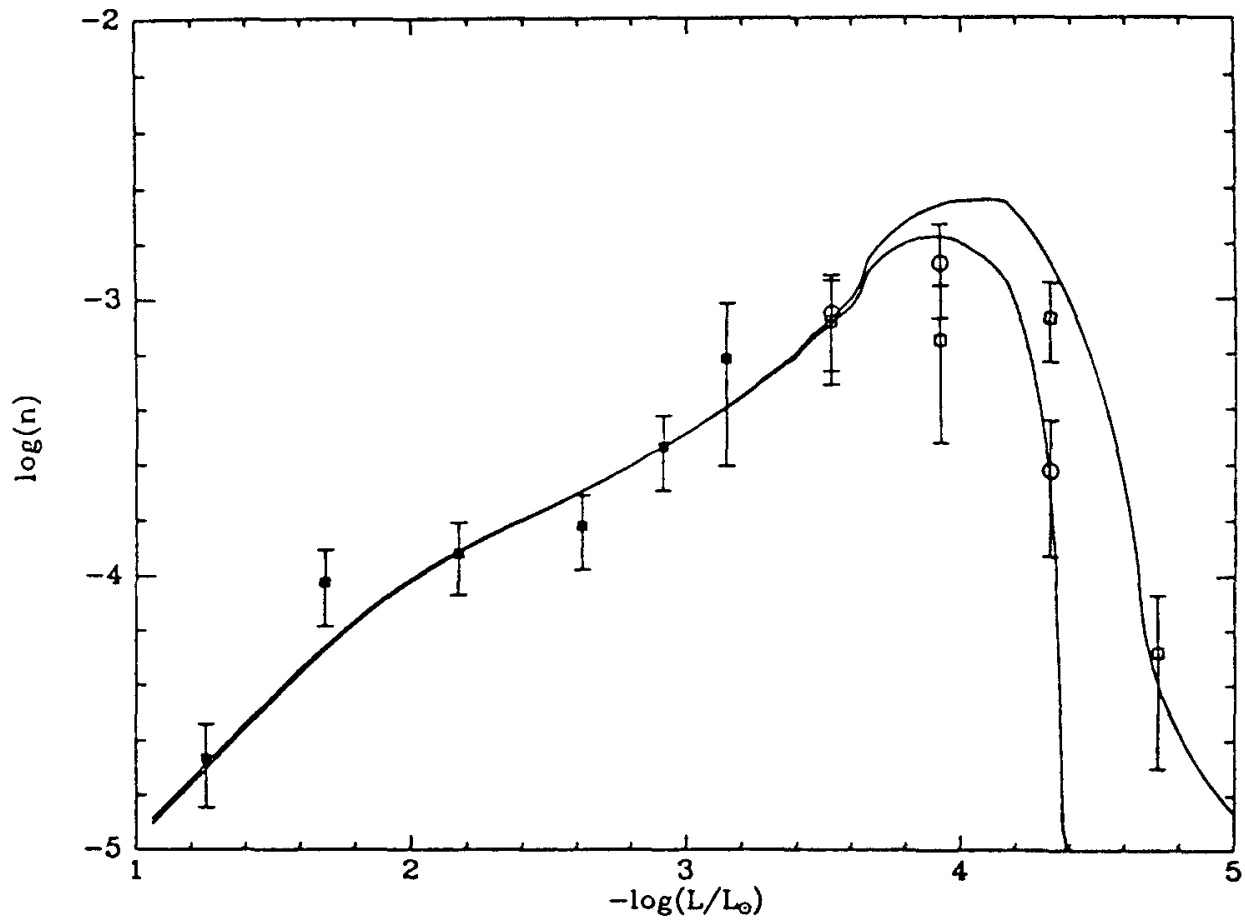

Fig. 8.10 Same as figure 9 but with an initial stratification for oxygen (Mazzitelli and D'Antona, 1986) - case (iii). The ages of the disk are respectively 8.5 (left) and 10.3 (right) Gyr.

the oxygen profile. The difference in the cooling time at $\log \left(L / L_{\odot}\right) \simeq-4.5$, and then in the estimate of the disk age, is about $0.5 \mathrm{Gyr}$.

When $\mathrm{C} / \mathrm{O}$ separation is taken into account, the ages of the disk are 10.5 and 12.3 Gyr for case (i) - figure 11 - and 9.5 and 12 Gyr for case (iv) - figure 12. The effect of initial stratification is slightly larger than for our reference model and case (iii), as could be expected from the corresponding cooling times. These calculations show that $\mathrm{C} / \mathrm{O}$ differentiation at crystallization increases the cooling time at the observed cut-off luminosity by 1.5 to $2 \mathrm{Gyr}$. Therefore, any estimate of the age of the galactic disk obtained without taking into account the $\mathrm{C} / \mathrm{O}$ differentiation process in the calculation of the luminosity function, must be increased by at least $1.5 \mathrm{Gyr}$.

The bump in the theoretical luminosity function at $\log \left(L / L_{\odot}\right) \simeq-4$ will be discussed later. The inclusion of selection effects due to the different scale heights of old and young white dwarfs leads to a reduction of the amplitude of this bump (see also Garcia-Berro et al 1988b for a detailed discussion).

We will now focus on the effect the crystallization of minor chemical elements and, in particular, on the effect of ${ }^{22} \mathrm{Ne}$. The corresponding luminosity function, computed according to equation (12) with the model of galactic chemical evolution already mentioned, is shown in figure 13 . In 


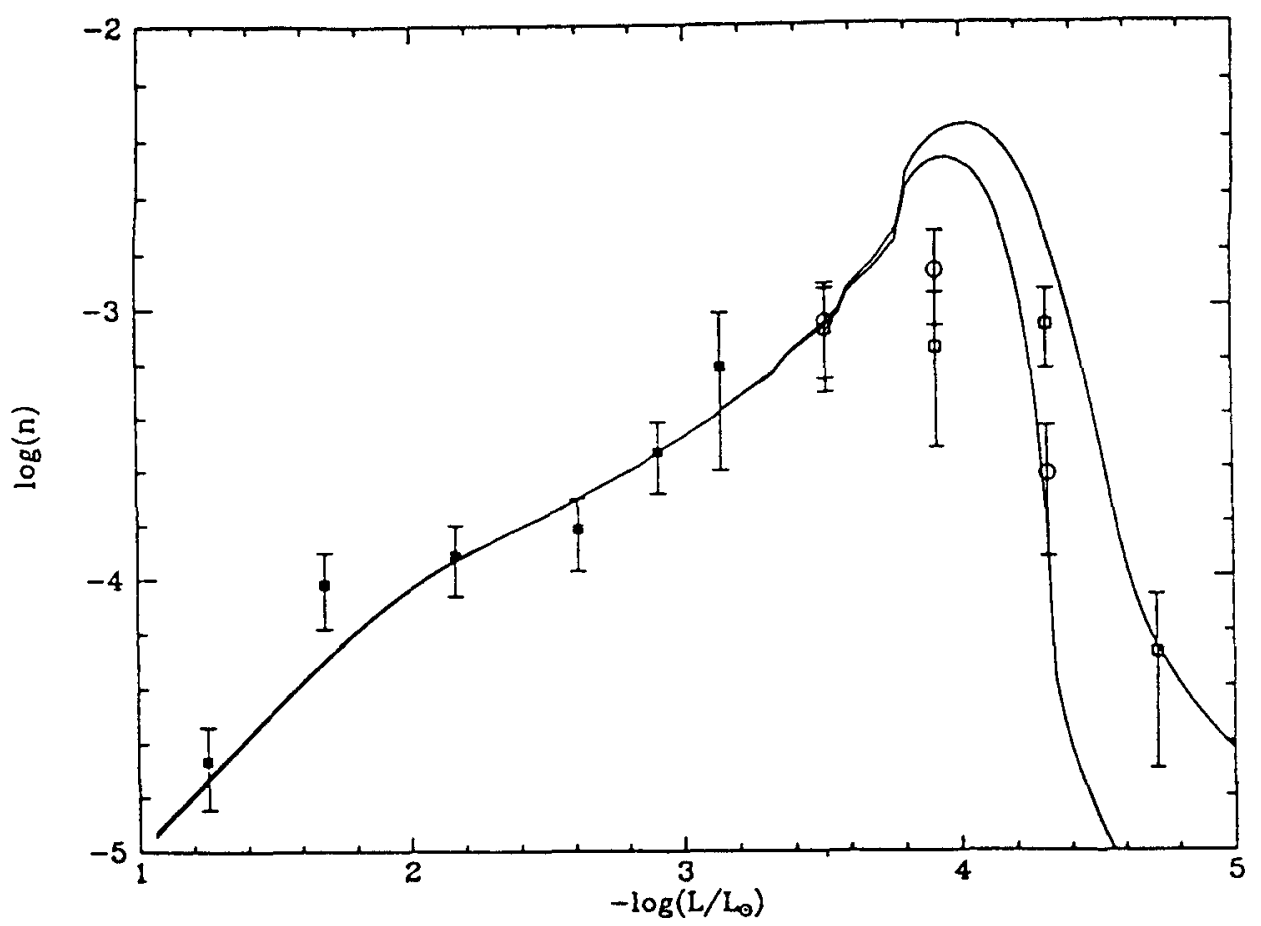

Fig. 8.11 Same as figure 9 but with $\mathrm{C} / \mathrm{O}$ fractionation - case (i). The ages of the disk are 10.5 and $12.3 \mathrm{Gyr}$.

order to characterize the effect of ${ }^{22} \mathrm{Ne}$ crystallization, we first ignore $\mathrm{C} / \mathrm{O}$ fractionation, and compare the results with our reference model (dotted line). The ages for the disk are again 8.8 and 10.5 Gyr. Therefore, although the cooling is strongly delayed by the presence of even a small amount of ${ }^{22} \mathrm{Ne}$, this effect is not reflected in the location of the cut-off of the white dwarf luminosity function.

This apparently surprising result stems from the strong dependence of the white dwarf luminosity function upon the shape of the age-metallicity relation. For standard models of chemical galactic evolution, the initial abundance of CNO cycle elements is zero and there is a gradual enrichment along the evolution of the galaxy, leading to a maximum for ${ }^{22} \mathrm{Ne}$ abundance of about $2-3 \%$ per mass, depending on the adopted yields. The stars old enough to reach the cut-off come from progenitors formed with the galaxy, with no ${ }^{22} \mathrm{Ne}$ enrichment. Therefore the age of the disk derived from the location of the cut-off is not affected by the presence of minor chemical species. For this reason, the effect of ${ }^{56} \mathrm{Fe}$ on the luminosity function, qualitatively quite similar to the effect of ${ }^{22} \mathrm{Ne}$, has not been considered explicitly. Its effect on the cooling time has been calculated in our previous paper (Segretain et al, 1993). This result demonstrates the impor- 


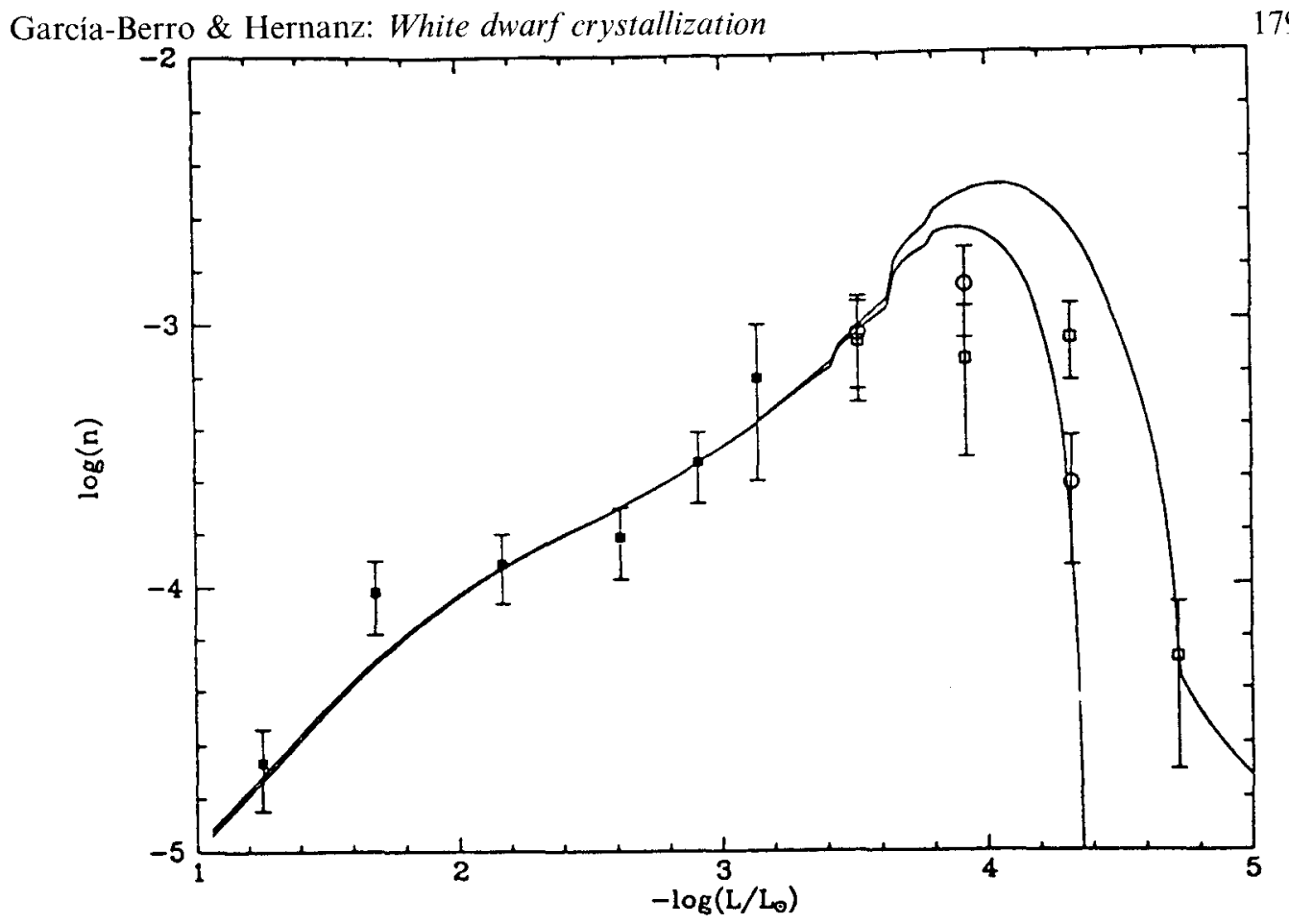

Fig. 8.12 Same as figure 9 but with an initial stratitication tor oxygen (Mazzitelli and D'Antona, 1986) - case (iv). The ages of the disk are 9.5 and 12 Gyr.

tance of a careful computation of the white dwarf luminosity function, with a complete model of galactic chemical evolution, and shows that the delays in the cooling times do not imply automatically increases of the disk age, as was the case for pure carbon-oxygen white dwarfs.

For a sake of completeness, we have also computed white dwarf luminosity functions for different initial metallicities of the galaxy. In figure 14 we compare the results for $Z_{0}=0$ and $Z_{0}=0.01$, for $t_{\text {disk }}=10 \mathrm{Gyr}$. A nonzero initial metallicity may reflect an initial metal enrichment of the galaxy due to a previous (Population III?) generation of massive stars. The cutoff position is shifted to higher luminosities for higher initial metallicity, as expected. For $Z_{0}=0.01$, the age of the disk must be increased by about 3 Gyr in order to reproduce the observed cut-off at the right luminosity.

Our last calculation corresponds to the most general case. This model takes into account both ${ }^{22} \mathrm{Ne}$ crystallization, and then crystallization of the remaining $\mathrm{C} / \mathrm{O}$ mixture, as given by the phase diagrams described in Segretain et al. (1993). Even though such calculation remains slightly uncertain from the quantitative viewpoint, because of the lack of an exact treatment of the crystallization diagram of three-component mixtures, it certainly retains most of the physical process, and then gives reasonable estimates. The 


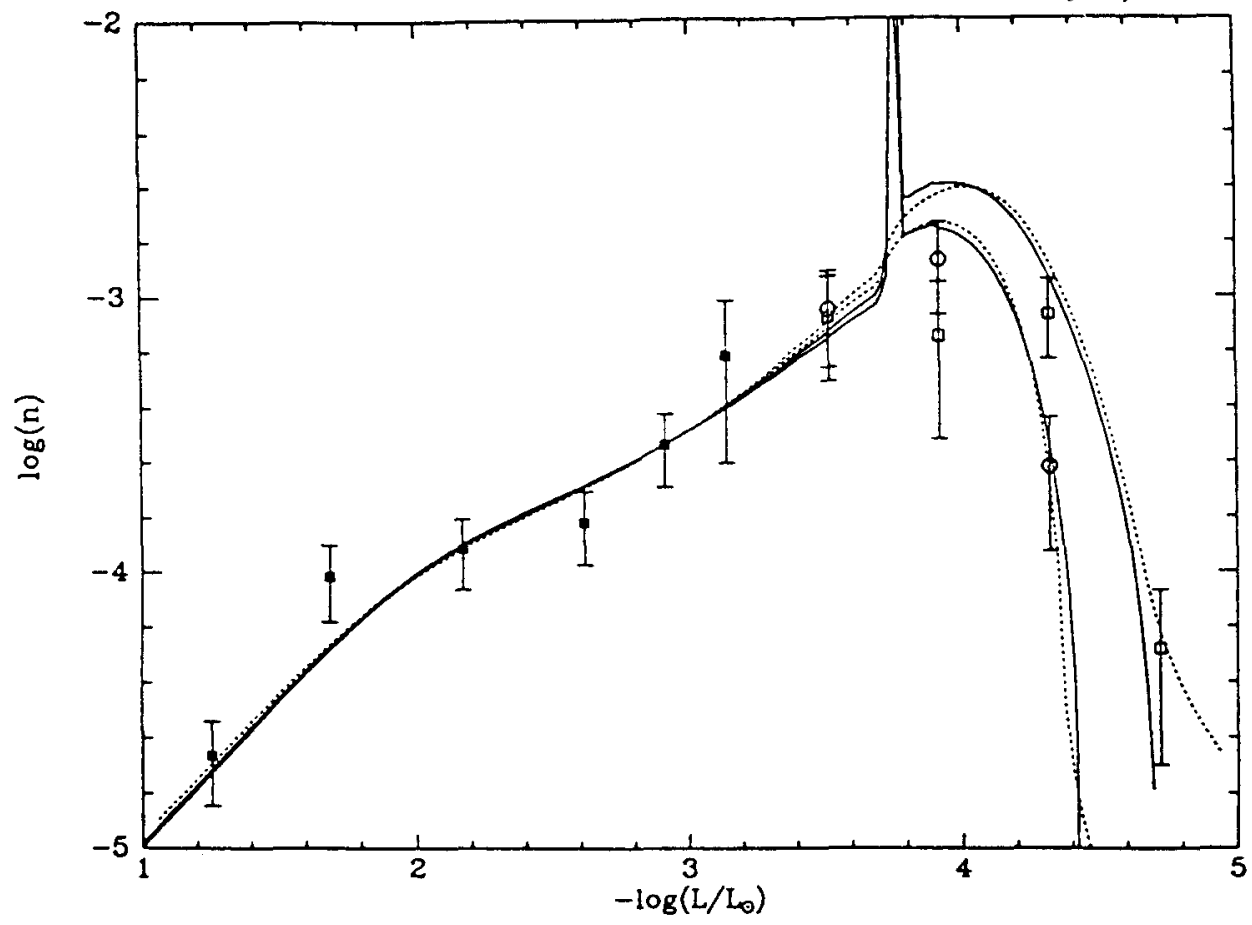

Fig. 8.13 Theoretical luminosity functions for $\mathrm{C} / \mathrm{O} / \mathrm{Ne}$ white dwarfs, taking into account ${ }^{22} \mathrm{Ne}$ deposition at crystallization (solid lines). Luminosity functions ignoring this process (reference model) are also shown for comparison (dotted lines). The ages of the disk are the same as in figure 9 (8.8 and $10.3 \mathrm{Gyr}$ ).

corresponding luminosity functions are shown in figures 15 and 16 (dotted lines), for disk ages 10.5 and 13 Gyr respectively, and are compared with the observed luminosity functions, obtained with the two approximations for the bolometric correction of cool white dwarfs (Liebert et al, 1988): blackbody bolometric correction for non DAs and a correction based on model atmospheres for DAs (figure 15) or zero bolometric correction for all cool white dwarfs (figure 16). The ages of the disk are very similar to the results of case (ii), i.e. zero metallicity, as expected. It is worth noting that the bump around $\log \left(L / L_{\odot}\right) \simeq-3.8$ is larger than for carbon-oxygen separation only.

We will now discuss the presence of a spike in the luminosity function (see figures 13-16) at $\log \left(L / L_{\odot}\right) \simeq-3.8$. This pattern is due to ${ }^{22} \mathrm{Ne}$ sedimentation, and its associated release of gravitational energy at a given luminosity, and could be used as the observable signature of this process. However, this has to be taken very cautiously for several reasons. First, these luminosity functions have been computed from equation (12), which considers that cooling times are those of a $0.6 \mathrm{M}_{\odot}$ white dwarf with different ${ }^{22} \mathrm{Ne}$ abundances, thus neglecting the effect of the mass of the white dwarf 


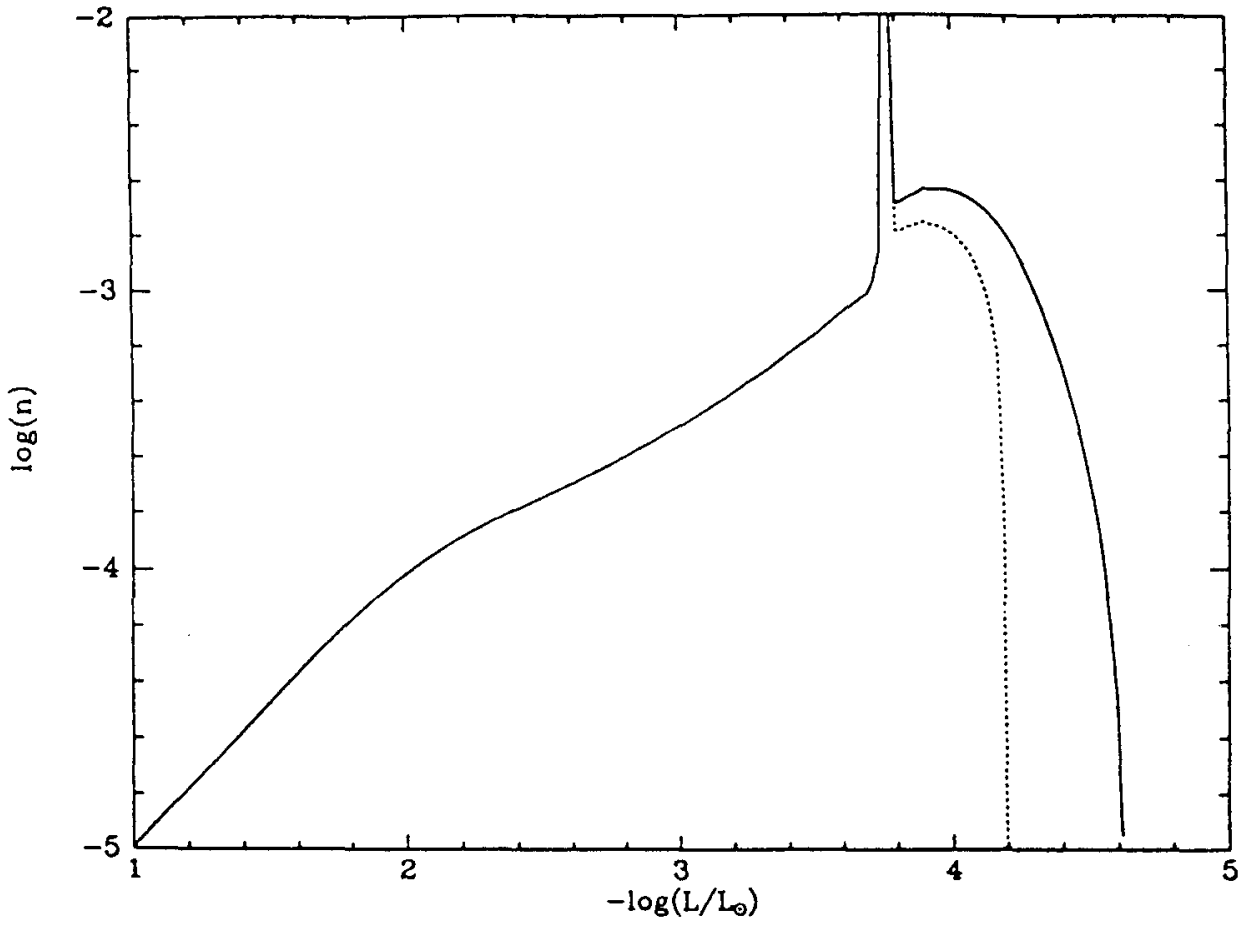

Fig. 8.14 Same as figure 13 for different initial metallicities of the galaxy: $\mathrm{Z}_{0}=0$ (solid line) and $\mathrm{Z}_{0}=0.01$ (dotted line), and $t_{d i s k}=10 \mathrm{Gyr}$.

on cooling. If the luminosity function were calculated for the whole white dwarf mass distribution, the spike would be spread over a larger range of luminosities, yielding a smoother luminosity function. Second, as already mentioned, old white dwarfs have larger scale heights over the galactic plane than young ones. This leads to a further reduction of the amplitude of the bump and the spike. This last effect has been included in our last calculation (see García-Berro et al, 1988b for details on the adopted scale height-age law). The corresponding luminosity functions are shown in figures 15 and 16 (solid lines). Furthermore, from an observational point of view, white dwarfs are actually binned in magnitude intervals. This must be taken into account for a correct comparison of theoretical and observational luminosity functions. Since the observed luminosity function is obtained by binning the data in bins of a certain amplitude in visual magnitude (usually, $\Delta M_{v}=0.5$ or 1 magnitude), we have followed the same procedure with the theoretical luminosity functions. We have averaged our results in bins of a certain amplitude, following the prescriptions of Liebert et al: $\Delta M_{v}=0.5$ for hot white dwarfs and $\Delta M_{b o l}=1$ for the cool ones, with the same relationship between $M_{b o l}$ and $M_{v}$ for hot white dwarfs and the two already mentioned possibilities for the bolometric corrections of cool white dwarfs. The results 


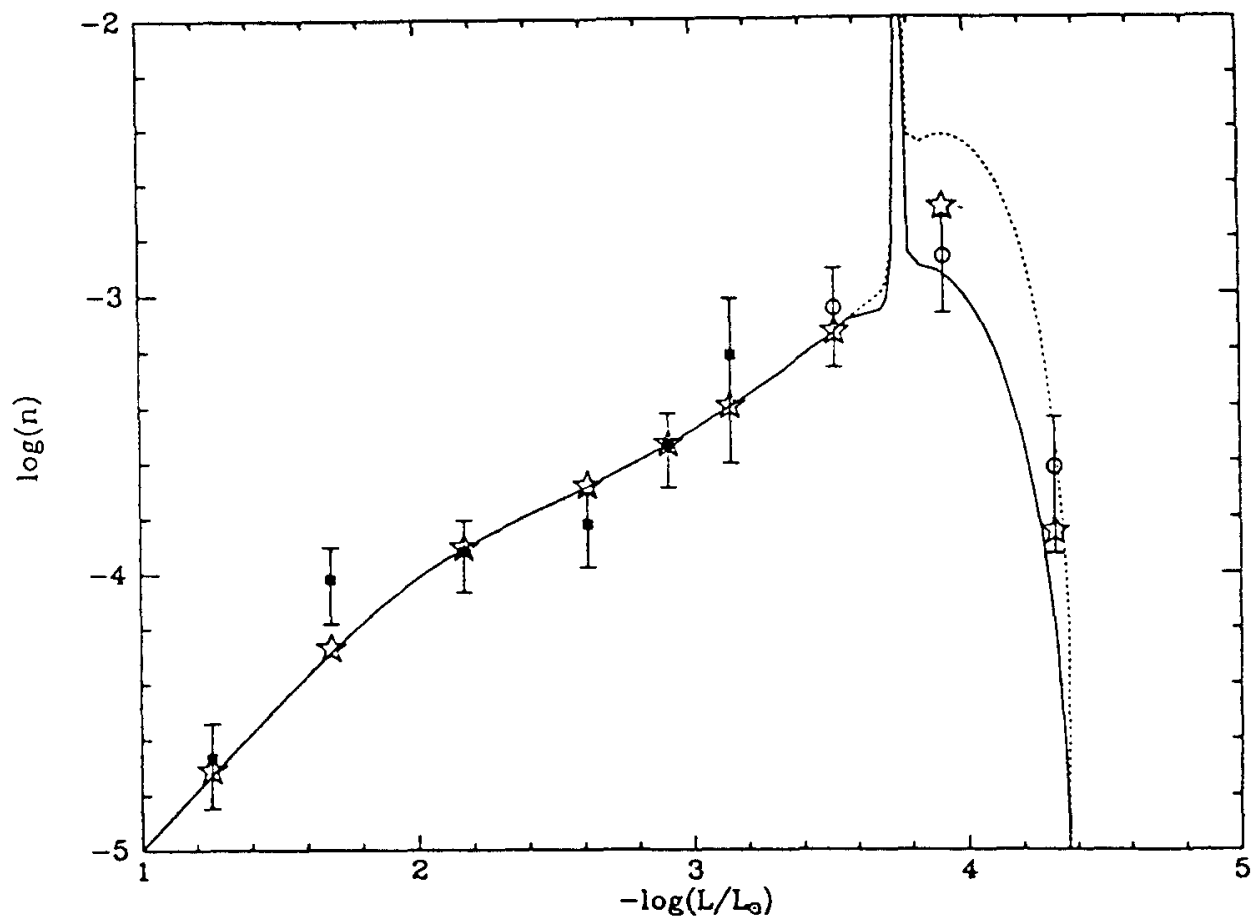

Fig. 8.15 Theoretical luminosity functions when both ${ }^{22} \mathrm{Ne}$ crystallization and then $\mathrm{C} / \mathrm{O}$ crystallization are taken into account successively. The effect of scale height inflation is either included (solid line) or ignored (dotted line), for $t_{\text {dist }}=10.5 \mathrm{Gyr}$. The observational points for cool white dwarfs are obtained with blackbody BCs for non DAs and model BCs for cool DAs. The open asterisks are the values of the binned theoretical luminosity function (solid line) (see text for details).

are shown by the asterisks in figures 15 and 16 , for our most general case. The larger discrepancy at $\log \left(L / L_{\odot}\right) \simeq-4$ for $t_{\text {disk }}=13$ Gyr (figure 16) is due to the much lower position of the observational point at this luminosity, when a zero bolometric correction is used. It is clear that a better knowledge of the faint end of the observed luminosity function, as well as a more complete treatment of the scale height problem, are crucial to withdraw reliable information on the age of the galactic disk.

\subsection{Conclusions}

We have examined the modification of the cooling time of old white dwarfs, due to the energy release associated with the chemical separation of major and minor elements at crystallization. Our calculations include up-to-date equations of state as well as accurate crystallization diagrams. The cooling time is computed directly from the temperature dependence of the binding energy, taking into account automatically the gravitational energy and the 


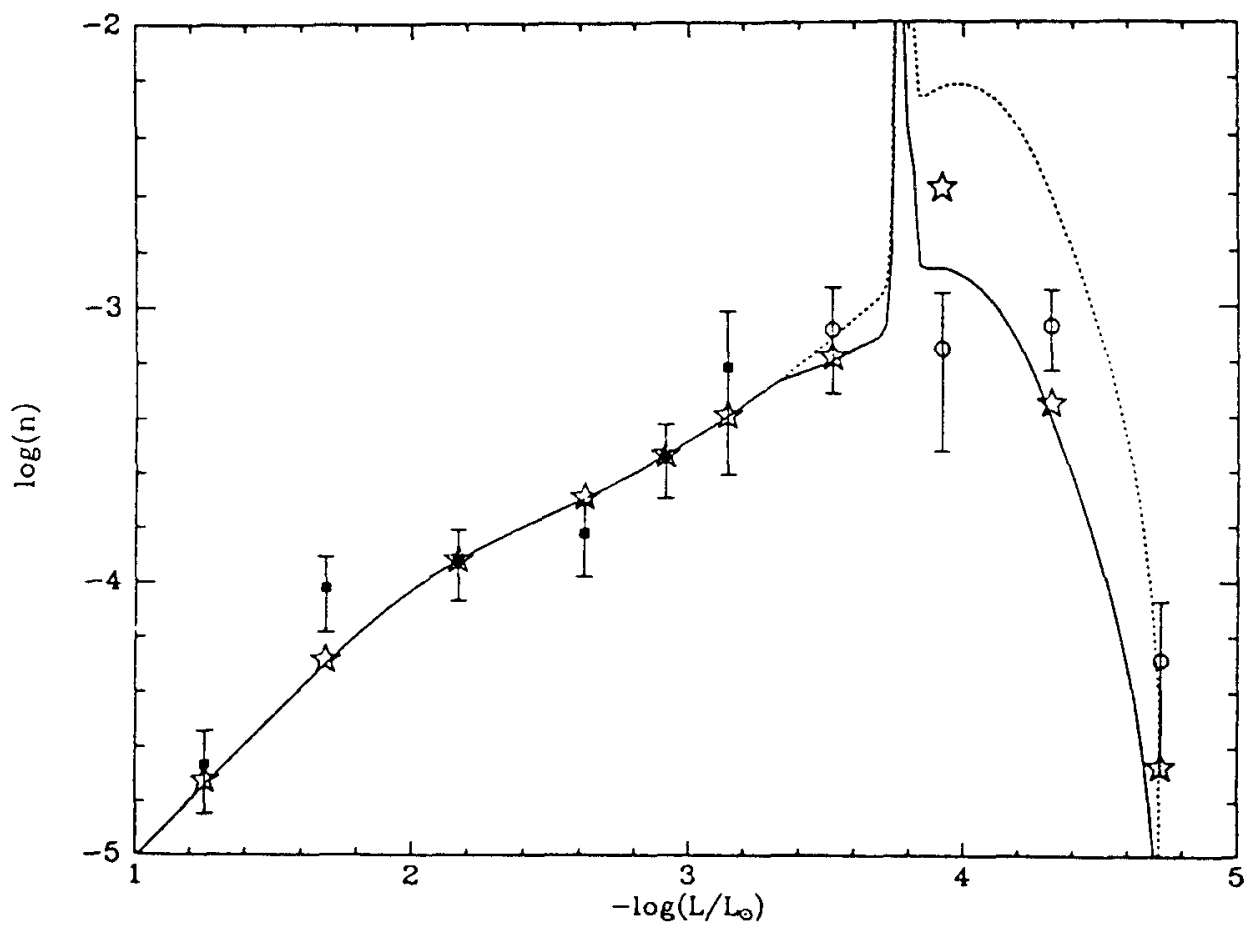

Fig. 8.16 Same as figure 15 but for $t_{d i s k}=13 \mathrm{Gyr}$. This age is obtained when fitting the observations corresponding to zero $\mathrm{BC}$ for cool white dwarfs.

latent heat released by crystallization along the evolution of the star. Our calculations consider also the possibility of an initial composition gradient in the $\mathrm{C}$ and $\mathrm{O}$ distributions.

We believe these calculations to provide the best estimate for the time delay induced by the crystallization of the $\mathrm{C} / \mathrm{O}$ mixture, a long time standing problem, and trace elements. We show that crystallization of minor elements has a drastic effect on the cooling time of the star, in spite of their low cosmic abundance. The energy released by crystallization of these elements produces a substantial extension of the cooling time, of the order or even larger than the one induced by the crystallization of $\mathrm{C} / \mathrm{O}$ itself, which is between 1 and $2 \mathrm{Gyr}$, depending on whether initial stratification is considered or not. Even though the exact determination of these processes would require the knowledge of a four-component phase diagram, our results demonstrate convincingly the importance of the crystallization of major and minor species in modern white dwarf cooling theory. The present calculations suggest that ${ }^{22} \mathrm{Ne}$ crystallizes first, in a homogeneous $\mathrm{C} / \mathrm{O}$ liquid, all ${ }^{22} \mathrm{Ne}$ being collected ultimately into the core, which yields a time delay of about $3 \mathrm{Gyr}$. Our treatment of the crystallization of the remaining $\mathrm{C} / \mathrm{O} /{ }^{56} \mathrm{Fe}$ mixture is certainly oversimplified, specially since we 
consider that the $\mathrm{C}, \mathrm{O}$ and ${ }^{56} \mathrm{Fe}$ abundances remain unchanged after ${ }^{22} \mathrm{Ne}$ crystallization occurs, and we ignore a possible ${ }^{56} \mathrm{Fe}$ phase separation in the liquid phase. For this reason, our calculations provide probably an upper limit for the time delay induced by crystallization of the afore-mentioned three-component-mixture. However, we can reasonably estimate this delay to be at least $1 \mathrm{Gyr}$. Therefore, if minor elements are present in white dwarf interiors, the minimum extension of the cooling age of a $0.6 M_{\odot}$ white dwarf could be as large as $4 \mathrm{Gyr}$, a fairly important amount.

On the other hand, theoretical luminosity functions have been also computed for pure $\mathrm{C} / \mathrm{O}$ white dwarfs and for white dwarfs with a chemical composition which includes minor chemical species such as ${ }^{22} \mathrm{Ne}$ and ${ }^{56} \mathrm{Fe}$. The calculations take into account the variation of metallicity along the chemical evolution of the Galaxy. We believe that these calculations give the most complete treatment of the effect of the different crystallization processes occuring in white dwarf interiors on the cooling history and the luminosity function of these stars. The theoretical luminosity functions are compared with the observed ones to determine the age of the galactic disk. We have shown that the time delay due to $\mathrm{C} / \mathrm{O}$ fractionation at crystallization directly translates into the luminosity function, and that any age of the disk obtained from calculations which do not include this effect must be increased by at least $1.5 \mathrm{Gyr}$. This gives $t_{\text {disk }} \sim 9.5$ to $12 \mathrm{Gyr}$, if we take Wood's results as reference. Although the presence of minor chemical species is found to modify appreciably the cooling time, this does not affect the age of the disk obtained from the luminosity function, a direct consequence of the low-metallicity of old white dwarfs. On the other hand, the important release of gravitational energy associated with the sedimentation of minor species produces a sharp peak in the luminosity function around $\log \left(L / L_{\odot}\right) \sim-3.6$. Though not detectable with present day observations, the presence of this peak provides a future observational test of the effect of minor chemical species on the cooling history of white dwarfs. If the initial metallicity of the Galaxy is non-zero, then the presence of minor chemical species modifies significantly the position of the cut-off and increases the age of the disk by about $3 \mathrm{Gyr}$ for $Z_{0}=1 \%$. These calculations demonstrate the necessity to include a proper treatment of crystallization in modern white dwarf cooling theory, and show convincingly the importance of galactic evolution for a proper determination of the age of the disk, stressing the need for further study in this direction. 


\section{References}

Abia, C., Canal, R., Isern, J., Ap. J, 366, 198, (1991)

Abrikosov A.A., Zh. Eksp. i Teor. Fiz., 39, 1798 (Soviet Phys. JETP 12, 1254), (1960)

Clayton, D.D., Ap. J, 285, 411, (1984)

Barrat J.L., Hansen J.P., Mochkovitch R., 1988, As. Astr., 199, L15, (1988)

Bravo, E., Isern, J., Canal, R., As. Ast., 270, 288, (1993)

Chabrier, G., Segretain, L., Hernanz, M., Isern, J., Mochkovitch, R. in White Dwarfs: Advances in Observation and Theory (Dordrecht: Kluwer Academic publishers) in press, (1993)

D'Antona, F., Mazzitelli, I., Ap. J, 347, 934, (1989)

Fleming, T.A., Liebert, J., Green, R.F., Ap. J 308, 176, (1986)

Garcia-Berro, E., Hernanz, M., Isern, J., Mochkovitch, R., Nature 333, 642, (1988a)

García-Berro, Hernanz, M., Mochkovitch, R., Isern, J., As. Astr., 193, 141, (1988b)

Iben, I., Laughlin, G., $A p . J, 341,312$, (1989)

Iben, I., Tutukov, A.V., Ap. J, 282, 615, (1984)

Iyetomi H., Ogata S., Ichimaru S., Ap. J 297, L17, (1988)

Isern, J., García-Berro, E., Hernanz, M., Mochkovitch, R., White Dwarfs (Berlin: Springer Verlag), 278, (1989a)

Isern, J., García-Berro, E., Hernanz, M., Mochkovitch, R., Evolutionary Phenomenae in Galaxies (Cambridge: Cambridge University Press), 224, (1989b)

sern, J., Mochkovitch, R., García-Berro, E., Hernanz, M., A. Astr., 241, L29, (1991)

Kirshnitz D.A., Zh. Eksp. i Teor. Fiz., 38, 503 (Soviet Phys. JETP 11, 365), (1960)

Lamb D.Q., Van Horn H.M., Ap. J., 200, 306, (1975)

Liebert, J., Dahn, C.C., Monet, D.G., Ap. J 332, 891, (1988)

Mazzitelli, I., D'Antona, F., Ap. J, 308, 706, (1986)

Mestel L., MNRAS 112, 583, (1952)

Mochkovitch R., As. Astr. 122, 212, (1983)

Monet, D.G., Dahn, C.C., Vrba, F.J., Harris, H.C., Pier, J.R., Luginbuhl, C.B., Ables, H.D., $A J, 103,638,(1992)$

Noh, H.R., Scalo, J., Ap. J, 352, 605, (1990)

Salpeter E.E., 1961, Ap. J 134, 669, (1961)

Segretain, L., Chabrier, G., As. Astr., 271, L13, (1993)

Stevenson D.J., J.Phys. Suppl. 41, C2-53, (1980)

Van Horn H.M., Phys. Lett. A, 28, 706, (1969)

Winget, D.E., Hansen, C.J., Liebert, J., Van Horn, H.M., Fontaine, G., Nather, R.E., Kepler, S.O., Lamb, D.Q., Ap. J, 315, L77, (1987)

Wood M.A., Ap. J 386, 536, (1992)

Wood, M.A., Winget, D.E., in IAU Colloq. 114, White Dwarfs (Berlin:

Springer-Verlag), 282, (1989)

Xu, Z.M., VanHorn, H.M., Ap. J, 387, 662, (1992)

Yuan, J.W., As. Astr., 224, 108, (1989)

Yuan, J.W., As. Astr., 261, 105, (1992) 